\title{
MAXIMUM AND MINIMUM FREE ENERGIES FOR A LINEAR VISCOELASTIC MATERIAL
}

\author{
$\mathrm{BY}$ \\ M. FABRIZIO (Dipartimento di Matematica, Università di Bologna, Bologna, Italia) \\ AND \\ J. M. GOLDEN (School of Mathematics, Statistics and Computer Science, Dublin Institute of \\ Technology, Dublin, Ireland)
}

\begin{abstract}
Certain results about free energies of materials with memory are proved, using the abstract formulation of thermodynamics, both in the general case and as applied within the theory of linear viscoelasticity. In particular, an integral equation for the strain continuation associated with the maximum recoverable work from a given linear viscoelastic state is shown to have a unique solution and is solved directly, using the Wiener-Hopf technique. This leads to an expression for the minimum free energy, previously derived by means of a variational technique in the frequency domain. A new variational method is developed in both the time and frequency domains. In the former case, this approach yields integral equations for both the minimum and maximum free energies associated with a given viscoelastic state. In the latter case, explicit forms of a family of free energies, associated with a given state of a discrete spectrum viscoelastic material, are derived. This includes both maximum and minimum free energies.
\end{abstract}

1. Introduction. Certain results about free energies of materials with memory are proved in this paper, using the abstract development of thermodynamics due to Noll [1] and Coleman and Owen [2], both in the general case and as formulated within the theory of linear viscoelasticity. These developments build on the results of Fabrizio, Giorgi and Morro [3]. Also, explicit forms of a family of free energies associated with a given state (in the sense of Noll [1]) of a linear viscoelastic material are given on the frequency domain, based on a generalisation of the variational approach of Golden [4], who derived a general expression for the isothermal minimum free energy of a linear viscoelastic material in the case of a scalar constitutive relation. A generalization of the developments reported in

Received September 1, 2000.

2000 Mathematics Subject Classification. Primary 74A15, 74D05, 74D10; Secondary 45E10, 30E20.

Our thanks go to G. Gentili for useful discussions on aspects of this work. This work has been supported by Italian M.U.R.S.T. through the 40th project "Mathematical Methods for Materials Science" and by a grant under the Enterprise Ireland International Collaboration Programme (1998).

E-mail address: fabrizio@dm.unibo.it

E-mail address: jmgolden@maths1.kst.dit.ie

(C)2002 Brown University 
[4] to the full tensorial case has been given recently by Deseri, Gentili and Golden [5], the results of which are also useful in the present work.

The paper is in two parts, A and B. In Part A, certain general theorems are proved. Where linear constitutive relations are considered, they are the full tensorial equations for a viscoelastic solid. In Part B, an extended variational technique is introduced in both the time domain and the frequency domain. In the latter case, explicit results are obtained in the case of a scalar constitutive relationship for a viscoelastic solid.

In Part A, a general definition is given for the minimum free energy associated with a state, and it is related to the maximum recoverable work (see also Day [6]). The concept of a minimal state is introduced, coinciding with the definition of a state in Noll's theory [1], also used in the recent work of Del Piero and Deseri $[8,9]$. It is shown that the minimum free energy is independent of the definition of state and, in particular, can be represented as a functional of the minimal state. A definition of the maximum free energy is given. This depends in general on the definition of state adopted, although with appropriate restrictions, it is a function of the minimal state.

Also, for a linear constitutive relation, the Wiener-Hopf equation introduced by Breuer and Onat [10] is studied. This is an equation for the strain continuation associated with the maximum recoverable work from a given state, a quantity directly related to the minimum free energy, as noted earlier. A uniqueness and existence theorem is proved for this equation. Also, it is solved directly, using the Wiener-Hopf technique. This in turn leads to an explicit formula for the minimum free energy in the full tensorial case, which generalizes the scalar result in [4] and agrees with the formula of Deseri, Gentili and Golden [5], derived by the same variational approach as in [4]. A generalization of the solution is also given which extends its domain of definition to that of the stress functional. Finally, by means of a norm related to the minimum free energy, it is possible to introduce a topology in the space of processes and to obtain the closure of this space, containing all relevant processes.

In Part B, a new variational approach is developed, firstly in the time domain, for which integral equations are determined for the continuation/history yielding minimum/maximum free energies. The former equation is of course the Wiener-Hopf equation of Breuer and Onat [10], studied in Part A. Certain other results are proved but explicit formulae are not obtained. The purpose of the time domain considerations is to elucidate the frequency domain treatment developed in the remaining sections. In the frequency domain, the new variational approach is essentially a generalization of the method of [4]. Explicit expressions are derived for a partially ordered family of free energies associated with a minimal state, in the case of discrete spectrum materials (those with a relaxation function given by a finite sum of decaying exponentials - also considered in some detail in [4]). Included in this family is the minimum free energy, already known, and the maximum free energy.

As noted earlier, the explicit frequency domain results in Part B are obtained for the scalar case. The generalization to the tensor case will be presented in a forthcoming paper. The time domain considerations in Part B are presented in full tensorial form.

The following notation will be used. Vectors are indicated by boldface characters. Tensors and matrices are denoted by boldface capitals. Sym is the set of all symmetric 
second-order tensors. Lin(Sym) is the set of all linear transformations from Sym to Sym. If $\mathbf{L}, \mathbf{M} \in \operatorname{Lin}$, then $\mathbf{L} \cdot \mathbf{M}$ stands for $\operatorname{tr}\left(\mathbf{L M}^{\top}\right)$. Also, $|\mathbf{L}|^{2}=\mathbf{L} \cdot \mathbf{L}$.

The reals are denoted by $\mathbb{R}$, the nonnegative reals by $\mathbb{R}^{+}$and the strictly positive reals by $\mathbb{R}^{++}$, with similar definitions of $\mathbb{R}^{-}, \mathbb{R}^{--}$.

\section{Part A: GENERAL THEOREMS.}

2. Fading memory. Consider a continuous body $\mathcal{B}$, undergoing deformation such that a material point at $\mathbf{X}$ in the reference configuration is at position $\mathbf{x}=\mathbf{p}(\mathbf{X}, t)$ at time $t$. The deformation of the body is characterized by $\mathbf{F}(\mathbf{X}, t)=\nabla_{\mathbf{X}} \mathbf{p}(\mathbf{X}, t)$. The tensor field $\mathbf{L}(\mathbf{X}, t)=\nabla_{\mathbf{x}} \dot{\mathbf{p}}(\mathbf{X}, t)$ is the velocity gradient. The superimposed dot indicates a material time derivative. We have the identity

$$
\dot{\mathbf{F}}(\mathbf{X}, t)=\mathbf{L}(\mathbf{X}, t) \mathbf{F}(\mathbf{X}, t) .
$$

In the context of linear theories, we will use the (symmetric) strain tensor $\mathbf{E}$ defined as

$$
2 \mathbf{E}(\mathbf{X}, t)=\mathbf{F}^{\top}(\mathbf{X}, t) \mathbf{F}(\mathbf{X}, t)-\mathbf{I},
$$

where $\mathbf{I}$ is the unit tensor.

Let us denote by $\mathbf{T}$ the Cauchy stress tensor. Our constitutive assumption is that the symmetric tensor $\mathbf{T}(\mathbf{X}, t)$ is a specified functional on a suitable set of histories $\mathbf{F}^{t}(\mathbf{X})$, defined as:

$$
\mathbf{F}^{t}(\mathbf{X}, s)=\mathbf{F}(\mathbf{X}, t-s), \quad s \in \mathbb{R}^{+} .
$$

The restriction of $\mathbf{F}^{t}(\mathbf{X})$ to $s \in \mathbb{R}^{++}$, denoted by $\mathbf{F}_{r}^{t}(\mathbf{X})$, is termed the past history. Thus $\mathbf{F}^{t}(\mathbf{X})=\left(\mathbf{F}(\mathbf{X}, t), \mathbf{F}_{r}^{t}(\mathbf{X})\right)$.

The stress tensor is given by the functional:

$$
\mathbf{T}(\mathbf{X}, t)=\hat{\mathbf{T}}\left(\mathbf{F}(\mathbf{X}, t), \mathbf{F}_{r}^{t}(\mathbf{X})\right)=\hat{\mathbf{T}}\left(\mathbf{F}^{t}(\mathbf{X})\right) .
$$

The property of fading memory can be expressed through the (Volterra) principle of dissipation of hereditary action [11], which states: "the modulus of the variation of quantity [given by (2.2)], when $\mathbf{F}^{t}$ varies in any way..... in the interval $\left(-\infty, t_{1}\right)$ (with $\left.t_{1}<t\right)$ can be made as small as we please by taking the interval $\left(t_{1}, t\right)$ sufficiently large".

In order to give a more precise definition of this property of fading memory at a material point $\mathbf{X} \in \mathcal{B}$, it is necessary to consider the set $\mathcal{D}$ of the histories that make up the domain of definition of the functional (2.2). We suppose this set $\mathcal{D}$ has the following properties:

1. $\mathcal{D}=\operatorname{Lin}_{2} \times \mathcal{D}_{r}$, where $\operatorname{Lin}_{2}$ denotes the set of second-order tensors, and $\mathcal{D}_{r}$ is a set of past histories that contains the space $L^{\infty}\left(\mathbb{R}^{+}\right)$.

2. If $\mathbf{F}^{t}(\mathbf{X}) \in \mathcal{D}$, then the partly static history $\mathbf{F}_{\tau}^{t}$, associated with $\mathbf{F}^{t}(\mathbf{X})$, belongs to $\mathcal{D}$, where:

$$
\mathbf{F}_{\tau}^{t}(\mathbf{X}, s)=\left\{\begin{array}{cc}
\mathbf{F}(\mathbf{X}, t), & s \in[0, \tau), \\
\mathbf{F}^{t}(\mathbf{X}, t-s), & s \in[\tau, \infty) .
\end{array}\right.
$$

Thus $\tau$ is the duration of the static part of the history. 
Definition 2.1. A viscoelastic material is characterized by the constitutive equation (2.2), where $\mathbf{F}^{t} \in \mathcal{D}$ and there exists a constitutive equation $\mathbf{T}(\mathbf{X}, t)=\tilde{\mathbf{T}}(\mathbf{F}(\mathbf{X}, t))$ of an elastic material, such that:

$$
\lim _{\tau \rightarrow \infty} \hat{\mathbf{T}}\left(\mathbf{F}_{\tau}^{t}(\mathbf{X})\right)=\tilde{\mathbf{T}}(\mathbf{F}(\mathbf{X}, t))
$$

Moreover $\left(\hat{\mathbf{T}}\left(\mathbf{F}_{\tau}^{t}(\mathbf{X})\right)-\tilde{\mathbf{T}}(\mathbf{F}(\mathbf{X}, t))\right)$ belongs to $L^{2}\left(\mathbb{R}^{+}\right)$as a function of $\tau$.

This definition includes an expression of the fading memory property and now it will be considered in the context of the linear relation

$$
\mathbf{T}(\mathbf{X}, t)=\mathbf{G}_{0}(\mathbf{X}) \mathbf{E}(\mathbf{X}, t)+\int_{0}^{\infty} \mathbf{G}^{\prime}(\mathbf{X}, s) \mathbf{E}^{t}(\mathbf{X}, s) d s,
$$

where $\mathbf{E}$ is the infinitesimal stress tensor ${ }^{1}$, which takes the place of $\mathbf{F}$ in the linear theory. The quantites $\mathbf{G}_{0}, \mathbf{G}^{\prime}$ are fourth-order tensors. Henceforth, the dependence on $\mathbf{X}$ of the various quantities will not be explicitly included. The domain $\mathcal{D}$ consists of the set of pairs $\left(\mathbf{E}(t), \mathbf{E}_{r}^{t}\right)$, such that $\mathbf{E}(t) \in \operatorname{Sym}$ and $\mathbf{G}^{\prime} \mathbf{E}_{r}^{t} \in L^{1}\left(\mathbb{R}^{+}\right)$.

In the linear theory, $\mathcal{D}$ includes constant histories by property 1 . It follows that the kernel $\mathbf{G}^{\prime}$ belongs to $L^{1}\left(\mathbb{R}^{+}\right)$. Property 2 follows from property 1 , since if $\mathbf{G}^{\prime} \in L^{1}\left(\mathbb{R}^{+}\right)$, we conclude that $\mathbf{G}^{\prime} \mathbf{E}_{\tau}^{t} \in L^{1}\left(\mathbb{R}^{+}\right)$, where $\mathbf{E}_{\tau}^{t}$ is the partly static history associated with $\mathbf{E}^{t}$. Thus

$$
\lim _{\tau \rightarrow \infty} \hat{\mathbf{T}}\left(\mathbf{E}_{\tau}^{t}\right)=\mathbf{G}_{\infty} \mathbf{E}(t)
$$

where

$$
\mathbf{G}_{\infty}=\mathbf{G}_{0}+\int_{0}^{\infty} \mathbf{G}^{\prime}(s) d s .
$$

We sce that (2.3) represents a viscoelastic material with the fading memory property, according to Definition 2.1, since the right-hand side of (2.4) is the stress associated with an elastic material. The tensor $\mathbf{G}_{\infty}$ must be a positive definite tensor, for the same reason.

3. Simple material systems and dissipation principles. In this section, we consider certain dissipation principles and prove some theorems, adopting for greater generality the axiomatic formulation of the thermodynamics of simple material systems due to Noll [1] and Coleman and Owen [2].

A map $P:\left[0, d_{p}\right) \rightarrow \operatorname{Lin}_{2}$, piecewise continuous on the time interval $\left[0, d_{p}\right)$ and defined as $P(\tau)=\mathbf{L}(\tau), \tau \in\left[0, d_{p}\right)$, is said to be a kinetic process of duration $d_{p} \in \mathbb{R}^{+}$. The set $\Pi$ of all accessible processes $P$ satisfies the following properties:

i) if $P \in \Pi$, then its restriction $P_{\left[t_{1}, t_{2}\right)}$ to the interval $\left[t_{1}, t_{2}\right) \subset[0, \infty)$ belongs to $\Pi ;^{2}$

\footnotetext{
${ }^{1}$ The principle of material frame indifference has the consequence that the dependence of $\hat{\mathbf{T}}$ on $\mathbf{F}_{r}^{t}$ must in fact occur through an objective strain measure such as $\mathbf{E}$ given by (2.1), which may be approximated, in the linear case, by the infinitesimal strain tensor. This quantity will also be denoted by $\mathbf{E}$.

${ }^{2}$ In the following, the restriction $P_{[0: t)}$ will be denoted only by $P_{t}$.
} 
ii) if $P_{1}, P_{2} \in \Pi$, then $P_{1} * P_{2} \in \Pi$, where

$$
P_{1} * P_{2}(\tau)= \begin{cases}P_{1}(\tau), & \tau \in\left[0, d_{p_{1}}\right), \\ P_{2}\left(\tau-d_{p_{1}}\right), & \tau \in\left[d_{p_{1}}, d_{p_{1}}+d_{p_{2}}\right) .\end{cases}
$$

The process $P_{0}$ of zero duration is referred to as the zero process.

Following [1] and [2], we introduce the notion of state by means of the definition of a simple material element.

DEFINITION 3.1. A simple solid material element at any point is a set $\{\Pi, \Sigma, \hat{C}, \hat{\rho}, \hat{W}\}$ such that:

a: $\Pi$ is the family of all accessible kinetic processes,

b: $\Sigma$, the state space, is the set of states $\sigma$ of the system,

c: $\hat{C}: \Sigma \rightarrow \operatorname{Lin}_{2}$ maps any state $\sigma$ onto the current value of the deformation gradient $\mathbf{F}$,

d: the map $\hat{\rho}: \Sigma \times \Pi \rightarrow \Sigma$ is the evolution function, which transforms the state $\sigma_{1}$, under the process $P$ into $\sigma_{2}=\hat{\rho}\left(\sigma_{1}, P\right)$. Moreover, the mapping is such that:

1. $\hat{\rho}\left(\sigma, P_{1} * P_{2}\right)=\hat{\rho}\left(\hat{\rho}\left(\sigma, P_{1}\right), P_{2}\right), \quad \forall P_{1}, P_{2} \in \Pi, \quad \sigma \in \Sigma$,

2. $\hat{\rho}\left(\sigma, P_{0}\right)=\sigma$,

e: the map $\hat{W}: \Sigma \times \Pi \rightarrow \mathbb{R}$ is the work response, which to each state $\sigma$ and process $P$ assigns the corresponding work. Finally, there exists a function $\hat{\mathbf{T}}: \Sigma \times \Pi \rightarrow$ Sym, the stress response, in terms of which the work can be written as: ${ }^{3}$

$$
\hat{W}(\sigma, P)=\int_{0}^{d_{p}} \hat{\mathbf{T}}\left(\sigma, P_{t}\right) \cdot \mathbf{L}(t) d t .
$$

From the additivity property of integrals we have the following important property of the work:

$$
W\left(\sigma, P_{1} * P_{2}\right)=W\left(\sigma, P_{1}\right)+W\left(\hat{\rho}\left(\sigma, P_{1}\right), P_{2}\right) .
$$

For materials with fading memory the state at time $t$ will be defined as the current value and the past history of the deformation gradient:

$$
\sigma=\left(\mathbf{F}(t), \mathbf{F}_{r}^{t}\right)=\left(\mathbf{F}^{t}\right) .
$$

Incidentally, it is apparent by $(2.2)$ that $\hat{\mathbf{T}}$ is a state function, in that it depends on the state only.

Now we can introduce an equivalence relation on the space state $\Sigma$ by means of the following:

Definition 3.2. Two states $\sigma_{1}, \sigma_{2} \in \Sigma$ are equivalent if

$$
\begin{aligned}
\hat{C}\left(\sigma_{1}\right) & =\hat{C}\left(\sigma_{2}\right), \\
\hat{\mathbf{T}}\left(\sigma_{1}, P\right) & =\hat{\mathbf{T}}\left(\sigma_{2}, P\right)
\end{aligned}
$$

for all $P \in \Pi$.

\footnotetext{
${ }^{3}$ Strictly, this applies only to incompressible materials since we have omitted the density. However, this is irrelevant in the linear approximation, which is of primary interest here.
} 
Definition 3.2 satisfies the requirements for an equivalence relation, which we denote by $\mathcal{R}$. If the space state $\Sigma$ of a simple material contains equivalent states, then it is possible to build a new state space $\Sigma_{R}$, for the given material element, as the quotient of $\Sigma$ on $\mathcal{R}$. The elements $\sigma_{R}$ of $\Sigma_{R}$ will be called minimal states. It is not however necessary to use, for the given material element, the minimal state space $\Sigma_{R}$. It is correct also to use any notion of state that satisfies Definition 3.1.

It is easy to prove:

Proposition 3.1. If $\sigma_{1}$ and $\sigma_{2}$ are two equivalent states, then

$$
W\left(\sigma_{1}, P\right)=W\left(\sigma_{2}, P\right), \quad \forall P \in \Pi .
$$

The converse is also true if $\hat{C}\left(\sigma_{1}\right)=\hat{C}\left(\sigma_{2}\right)$.

Definition 3.3. A pair $(\sigma, P)$ is called a cyclic process if $\hat{\rho}(\sigma, P)=\sigma$.

For material elements with fading memory, cyclic processes $(\sigma, P)$ are realized only by a periodic history $\sigma=\mathbf{F}^{t}$, and by a process $P$ with duration equal to a finite integral number of periods of $\mathbf{F}^{t}$.

In this work, we are interested only in isothermal processes. For such processes the Second Law of Thermodynamics can be written (see [2]) as:

The Dissipation Principle: For every cyclic process $(\sigma, P)$ we have:

$$
W(\sigma, P)=\int_{0}^{d_{p}} \hat{\mathbf{T}}\left(\sigma, P_{t}\right) \cdot \mathbf{L}(t) d t \geq 0 .
$$

Definition 3.4. A set $\mathcal{S} \subset \Sigma$ is invariant under $\hat{\rho}$, if for every $\sigma_{1} \in \mathcal{S}$, and $P \in \Pi$, the state $\sigma=\hat{\rho}\left(\sigma_{1}, P\right) \in \mathcal{S}$.

Definition 3.5. A function $\psi: \mathcal{S}_{\psi} \rightarrow \mathbb{R}^{+}$is a free energy if :

i. the domain $\mathcal{S}_{\psi}$ is invariant under $\hat{\rho}$,

ii. for any pair $\sigma_{1}, \sigma_{2} \in \mathcal{S}_{\psi}$ and $P \in \Pi$, such that $\hat{\rho}\left(\sigma_{1}, P\right)=\sigma_{2}$ we have

$$
\psi\left(\sigma_{2}\right)-\psi\left(\sigma_{1}\right) \leq W\left(\sigma_{1}, P\right) .
$$

Let the set of all free energies of the simple material element under consideration be denoted by $\Psi$.

In Day [12], [6], Coleman and Owen [2], and Fabrizio, Giorgi and Morro [3], the existence of a free energy was proved as a consequence of the Dissipation Principle expressed by inequality (3.3). We consider here the problem of the existence of a minimum free energy and study its properties, building on the results of [3].

We define

$$
\mathcal{W}(\sigma)=\{W(\sigma, P) ; P \in \Pi\}
$$

The Strong Dissipation Principle. The set $\mathcal{W}(\sigma)$ is bounded below for all $\sigma \in \Sigma$. Furthermore, there is a state $\sigma^{\dagger}$, which we refer to as the zero state, such that

$$
\inf \mathcal{W}\left(\sigma^{\dagger}\right)=0
$$


REMARK 3.1. For a fading memory material, the zero state is $\sigma^{\dagger}=\mathbf{0}^{\dagger}$, where $\mathbf{0}^{\dagger}$ is the zero history

$$
\mathbf{F}^{t}(s)=0 \quad \forall s \in \mathbb{R}^{+} .
$$

Definition 3.6. A state $\sigma \in \Sigma$ is attainable from all of $\Sigma$ if, for any initial state $\sigma^{i}$, there exists a process $P \in \Pi$ such that $\hat{\rho}\left(\sigma^{i}, P\right)=\sigma$. A simple material system is attainable if any state $\sigma$ is attainable from every other state $\sigma^{\prime} \in \Sigma$.

It is shown in [3] that the Dissipation Principle follows from the Strong Dissipation Principle; also, the converse proposition holds if the system is attainable. However, for a simple material system with fading memory not all states are attainable. For this reason, in the following we adopt the Strong Dissipation Principle.

Let us define the set

$$
\Phi:=\left\{\phi: \Sigma \rightarrow \mathbb{R}^{+} ; \phi(\sigma) \leq \psi(\sigma) \forall \psi \in \Psi, \forall \sigma \in \mathcal{S}_{\psi} ; \phi\left(\sigma^{\dagger}\right)=0\right\}
$$

and the function $\phi_{M}: \Sigma \rightarrow \mathbb{R}^{+}$, where

$$
\phi_{M}(\sigma)=\sup \{\phi(\sigma) ; \phi \in \Phi\} .
$$

Thus $\phi_{M}$ is the largest functional with the property that it is less than or equal to any free energy for all states.

We define $\widetilde{\mathcal{W}}(\sigma)$ as

$$
\widetilde{\mathcal{W}}(\sigma):=\left\{W(\sigma, P)-\phi_{M}(\hat{\rho}(\sigma, P)) ; P \in \Pi\right\} .
$$

\section{Minimum and maximum free energies.}

DEFINITION 4.1. A functional $\psi_{m}$ is called the minimum free energy if:

i) $\psi_{m}$ is a free energy with domain $\mathcal{S}_{\psi}=\Sigma$,

ii) the zero state $\sigma^{\dagger} \in \Sigma$ is such that $\psi_{m}\left(\sigma^{\dagger}\right)=0$,

iii) for any free energy $\psi: \mathcal{S}_{\psi} \rightarrow \mathbb{R}^{+}$such that $\sigma^{\dagger} \in \mathcal{S}_{\psi}$ and $\psi\left(\sigma^{\dagger}\right)=0$, we have:

$$
\psi(\sigma) \geq \psi_{m}(\sigma) \forall \sigma \in \mathcal{S}_{\psi} .
$$

REMARK 4.1. The minimum free energy (if it exists) is unique. The proof follows easily from the inequality (4.1).

THEOREM 4.1. The functional

$$
\psi_{m}(\sigma):=-\inf \mathcal{W}(\sigma)
$$

is the minimum free energy.

The proof of this theorem is given in [3].

THEOREM 4.2. The functional

$$
\tilde{\psi}_{m}(\sigma):=-\inf \widetilde{\mathcal{W}}(\sigma)
$$

is a free energy such that $\tilde{\psi}_{m}(\sigma)=\psi_{m}(\sigma) \forall \sigma \in \Sigma$. 
Proof. By virtue of the Strong Dissipation Principle and the fact that $\phi_{M} \geq 0$, we see that it is well-defined for all $\sigma \in \Sigma$. We can write (4.2) in the alternative form

$$
\tilde{\psi}_{m}(\sigma)=\sup \left\{-W(\sigma, P)+\phi_{M}(\hat{\rho}(\sigma, P)) ; P \in \Pi\right\} .
$$

For the zero process $P_{0}, W\left(\sigma, P_{0}\right)$ vanishes and $\phi_{M I}\left(\hat{\rho}\left(\sigma, P_{0}\right)\right)$ is nonnegative. Thus $\tilde{\psi}_{m}(\sigma) \geq 0$. Note that $\psi_{m}\left(\sigma^{\dagger}\right)=0$.

Consider two states $\sigma_{1}, \sigma_{2}$ and a process $P^{\prime}$ such that $\hat{\rho}\left(\sigma_{1}, P^{\prime}\right)=\sigma_{2}$. It follows from (4.3) that for any $P \in \Pi$

$$
\tilde{\psi}_{m}\left(\sigma_{1}\right) \geq-W\left(\sigma_{1}, P^{\prime} * P\right)+\phi_{M}\left(\hat{\rho}\left(\sigma_{1}, P^{\prime} * P\right)\right)
$$

and that, for any $\varepsilon>0$, there is a process $P_{\varepsilon}$ such that

$$
\tilde{\psi}_{m}\left(\sigma_{2}\right)-\varepsilon<-W\left(\sigma_{2}, P_{\varepsilon}\right)+\phi_{M}\left(\hat{\rho}\left(\sigma_{2}, P_{\varepsilon}\right)\right) .
$$

Combining (4.4) and (4.5), we obtain

$$
\begin{aligned}
& \tilde{\psi}_{m}\left(\sigma_{2}\right)-\tilde{\psi}_{m}\left(\sigma_{1}\right)-\varepsilon \\
& \quad<-W\left(\sigma_{2}, P_{\varepsilon}\right)+\phi_{M}\left(\hat{\rho}\left(\sigma_{2}, P_{\varepsilon}\right)\right)+W\left(\sigma_{1}, P^{\prime} * P\right)-\phi_{M}\left(\hat{\rho}\left(\sigma_{1}, P^{\prime} * P\right)\right) \\
& \quad=-W\left(\sigma_{2}, P_{\varepsilon}\right)+\phi_{M}\left(\hat{\rho}\left(\sigma_{2}, P_{\varepsilon}\right)\right)+W\left(\sigma_{1}, P^{\prime}\right)+W\left(\sigma_{2}, P\right)-\phi_{M}\left(\hat{\rho}\left(\sigma_{2}, P\right)\right)
\end{aligned}
$$

by virtue of (3.2) and (3.1). Identifying $P_{\varepsilon}$ and $P$, the latter being arbitrary, we deduce that

$$
\tilde{\psi}_{m}\left(\sigma_{2}\right)-\tilde{\psi}_{m}\left(\sigma_{1}\right) \leq W\left(\sigma_{1}, P^{\prime}\right),
$$

which completes the proof that $\tilde{\psi}_{m}(\sigma)$ is a free energy. It remains to show that $\psi_{m}(\sigma)=$ $\tilde{\psi}_{m}(\sigma)$. Since $\phi_{M}$ is nonnegative, we have $W(\sigma, P) \geq W(\sigma, P)-\phi_{M}(\sigma) \forall P \in \Pi$ so that $\inf \mathcal{W}(\sigma) \geq \inf \widetilde{\mathcal{W}}(\sigma)$ or

$$
\psi_{m}(\sigma) \leq \tilde{\psi}_{m}(\sigma) \forall \sigma \in \Sigma .
$$

Given $\sigma \in \mathcal{S}_{\psi}$, for every $\varepsilon>0$, there is a process $P_{\varepsilon}$ such that

$$
\tilde{\psi}_{m}(\sigma)<-W\left(\sigma, P_{\varepsilon}\right)+\phi_{M}\left(\hat{\rho}\left(\sigma, P_{\varepsilon}\right)\right)+\varepsilon .
$$

Letting $\sigma_{\varepsilon}=\hat{\rho}\left(\sigma, P_{\varepsilon}\right)$, we have

$$
\psi\left(\sigma_{\varepsilon}\right)-\psi(\sigma) \leq W\left(\sigma, P_{\varepsilon}\right)
$$

for any free energy $\psi$. Combining these inequalities, we obtain

$$
\begin{aligned}
\tilde{\psi}_{m}(\sigma) & <-W\left(\sigma, P_{\varepsilon}\right)+\phi_{M I}\left(\sigma_{\varepsilon}\right)+\varepsilon \\
& \leq-\psi\left(\sigma_{\varepsilon}\right)+\psi(\sigma)+\phi_{M I}\left(\sigma_{\varepsilon}\right)+\varepsilon \\
& \leq \psi(\sigma)+\varepsilon
\end{aligned}
$$

since $\phi_{M}\left(\sigma_{\varepsilon}\right) \leq \psi\left(\sigma_{\varepsilon}\right)$ by definition. Thus, since $\varepsilon$ is arbitrary, we have that

$$
\tilde{\psi}_{m}(\sigma) \leq \psi(\sigma)
$$

for all $\psi$ and, in particular, for $\psi=\psi_{m}$. It follows from (4.6) and (4.8) that $\tilde{\psi}_{m}(\sigma)=$ $\psi_{m}(\sigma) \forall \sigma \in \Sigma$, which completes the proof. 
REMARK 4.2. A similar result holds for any choice of $\phi \in \Phi$ defined by (3.7). On examining the last part of the proof of Theorem 4.2, we can in fact weaken considerably the constraint on the functions $\phi \in \Phi$ that they be less than or equal to all free energies for all states.

Corollary 4.3. If $\exists \varepsilon_{0}>0$ such that for $\varepsilon<\varepsilon_{0}$,

$$
\phi_{M}\left(\hat{\rho}\left(\sigma, P_{\varepsilon}\right)\right) \leq \psi\left(\hat{\rho}\left(\sigma, P_{\varepsilon}\right)\right) \quad \forall \psi \in \Psi, \sigma \in \mathcal{S}_{\psi},
$$

where $P_{\varepsilon}$ is defined by (4.7), then Theorem 4.2 holds with $\widetilde{\mathcal{W}}(\sigma)$ defined by (3.9), although now $\phi_{M}$ is constrained only by (4.9) rather than by $(3.8)$.

Thus, in fact, the property must hold only for the final states of processes in the vicinity of the optimal process.

REMARK 4.3. It is always possible to represent the minimum free energy as a function of the minimal state $\sigma_{R}$. It is clear from the definition of $\mathcal{W}(\sigma)$, given by (3.5), and from the fact that $W(\sigma, P)=W\left(\sigma_{R}, P\right)$ for all $P \in \Pi$, that

$$
\inf \mathcal{W}(\sigma)=\inf \mathcal{W}\left(\sigma_{R}\right)
$$

Therefore, if $\sigma \in \sigma_{R}$,

$$
\psi_{m}(\sigma)=\psi_{m}\left(\sigma_{R}\right)
$$

Hence the minimum free energy is independent of the definition of state that is used.

Let us denote by $\Sigma_{\sigma}$ the set of all $\sigma^{\prime} \in \Sigma$ attainable from $\sigma$, viz.

$$
\Sigma_{\sigma}=\left\{\sigma^{\prime} \in \Sigma ; \quad \exists P \in \Pi ; \quad \sigma^{\prime}=\hat{\rho}(\sigma, P)\right\} .
$$

For any pair $\sigma_{0}, \sigma \in \Sigma$, such that $\sigma \in \Sigma_{\sigma_{0}}$, we can consider the set:

$$
N\left(\sigma_{0}, \sigma\right)=\left\{W\left(\sigma_{0}, P\right), \quad \forall P \in \Pi ; \hat{\rho}\left(\sigma_{0}, P\right)=\sigma\right\} .
$$

From the Strong Dissipation Principle, this set is bounded below.

TheOREM 4.4. For any fixed $\sigma^{i}$, the functional $\psi_{M}^{\sigma^{i}}: \Sigma_{\sigma^{i}} \rightarrow \mathbb{R}^{+}$defined by

$$
\psi_{M}^{\sigma^{i}}(\sigma)=\inf N\left(\sigma^{i} ; \sigma\right)+\psi_{m}\left(\sigma^{i}\right)
$$

is a free energy, called a maximal free energy. For any free energy $\psi: \mathcal{S}_{\psi} \rightarrow \mathbb{R}^{+}$, such that $\mathcal{S}_{\psi} \supset \Sigma_{\sigma^{i}}$, and $\psi\left(\sigma^{i}\right)=\psi_{m}\left(\sigma^{i}\right)$, we have

$$
\psi(\sigma) \leq \psi_{M}^{\sigma^{i}}(\sigma), \quad \forall \sigma \in \Sigma_{\sigma^{i}}
$$

Proof. The functional $\psi_{M}^{\sigma^{i}}$ is clearly well-defined on the set $\Sigma_{\sigma^{i}}$, which is invariant under $\hat{\rho}$. Let us consider a pair $\sigma_{1}, \sigma_{2} \in \Sigma_{\sigma^{i}}$ and a process $P \in \Pi$, such that $\sigma_{2}=\hat{\rho}\left(\sigma_{1}, P\right)$. For any $\varepsilon>0$, there exists a process $P_{1}^{\varepsilon}$ such that

$$
\hat{\rho}\left(\sigma^{i}, P_{1}^{\varepsilon}\right)=\sigma_{1}, \quad \psi_{M}^{\sigma^{i}}\left(\sigma_{1}\right)>W\left(\sigma^{i}, P_{1}^{\varepsilon}\right)+\psi_{m}\left(\sigma^{i}\right)-\varepsilon .
$$

Moreover,

$$
\psi_{M}^{\sigma^{i}}\left(\sigma_{2}\right) \leq W\left(\sigma^{i}, P_{1}^{\varepsilon} * P\right)+\psi_{m}\left(\sigma^{i}\right)
$$


Thus, by (4.13) and (4.14), we have

$$
\psi_{M}^{\sigma^{i}}\left(\sigma_{2}\right)-\psi_{M}^{\sigma^{i}}\left(\sigma_{1}\right) \leq W\left(\sigma_{1}, P\right) .
$$

Let $\psi: \mathcal{S}_{\psi} \rightarrow \mathbb{R}^{+}$be a free energy such that $\mathcal{S}_{\psi} \supset \Sigma_{\sigma^{i}}$, and $\psi\left(\sigma^{i}\right)=\psi_{m}\left(\sigma^{i}\right)=\psi_{M}^{\sigma^{i}}\left(\sigma^{i}\right)$. For any $\varepsilon>0$ there is a $P_{\varepsilon}$ such that $\hat{\rho}\left(\sigma^{i}, P_{\varepsilon}\right)=\sigma$ and $W\left(\sigma^{i}, P_{\varepsilon}\right)+\psi_{m}\left(\sigma^{i}\right)<\psi_{M}^{\sigma^{i}}(\sigma)+\varepsilon$. Also, since $\psi$ is a free energy, $\psi(\sigma) \leq W\left(\sigma^{i}, P_{\varepsilon}\right)+\psi\left(\sigma^{i}\right)=W\left(\sigma^{i}, P_{\varepsilon}\right)+\psi_{m}\left(\sigma^{i}\right)$. Since $\varepsilon$ is arbitrary the result follows.

REMARK 4.4. If for a given $\sigma^{i} \in \Sigma$ and a process $P$, a free energy $\psi(\sigma) \neq \psi_{M}(\sigma)$ (in particular $\left.\psi_{m}(\sigma)\right)$ obeys the relation

$$
\psi(\sigma)=W\left(\sigma^{i}, P\right)+\psi_{m}\left(\sigma^{i}\right)
$$

it follows that $P \notin \Pi$, although it may be the limit of a sequence of processes in $\Pi$. This situation in fact occurs for the minimum free energy given by the maximum recoverable work in the linear case discussed later (comment after (8.3)), and explains why the process producing the maximum recoverable work is singular.

REMARK 4.5. Of course for any $\sigma^{i} \in \Sigma$ we may obtain a different free energy. Moreover, for a fixed $\sigma^{i} \in \Sigma$ the definition of maximum free energy may depend on the definition of state. We can, however, construct a maximum free energy that is defined on the space of minimal states. In other words, if we consider the definition of minimal state, then (4.11) is replaced by

$$
N\left(\sigma_{0 R}, \sigma_{R}\right)=\left\{W\left(\sigma_{0 R}, P\right), \quad \forall P \in \Pi ; \hat{\rho}\left(\sigma_{0 R}, P\right)=\sigma_{R}\right\} .
$$

This set is generally larger than $N\left(\sigma_{0}, \sigma\right)$, if $\sigma_{0} \in \sigma_{0 R}$ and $\sigma \in \sigma_{R}$. For this reason the maximum free energy defined on $\Sigma_{R}$ as

$$
\psi_{M}^{\sigma_{R}^{i}}\left(\sigma_{R}\right)=\inf N\left(\sigma_{R}^{i} ; \sigma_{R}\right)+\psi_{m}\left(\sigma_{R}^{i}\right)
$$

satisfies the inequality

$$
\psi_{M}^{\sigma_{R}^{i}}\left(\sigma_{R}\right) \leq \psi_{M}^{\sigma^{i}}(\sigma), \quad \sigma^{i} \in \sigma_{R}^{i}, \quad \sigma \in \sigma_{R}
$$

Relation (4.12) will apply to any free energy $\psi\left(\sigma_{R}\right)$ defined on $\Sigma_{R}$ provided $\psi\left(\sigma_{R}^{i}\right)=$ $\psi_{m}\left(\sigma_{R}^{i}\right)$. We shall give the form of the maximum free energy $\psi_{M}^{\sigma_{R}^{i}}\left(\sigma_{R}\right)$ on $\Sigma_{R}$ for a particular linear model in Sec. 11.

5. Linear viscoelasticity. For the remainder of this paper, we shall be dealing with the case of a linear viscoelastic solid, characterized by the stress-strain relation (2.3). Ignoring $\mathbf{X}$ dependence, we write it in the form

$$
\mathbf{T}(t)=\mathbf{G}_{0} \mathbf{E}(t)+\int_{0}^{\infty} \mathbf{G}^{\prime}(s) \mathbf{E}^{t}(s) d s,
$$

where, having already concluded in Sec. 2 that $\mathbf{G}^{\prime}(\cdot) \in L^{1}\left(\mathbb{R}^{+}\right)$, now we shall further assume that $\mathbf{G}^{\prime}(\cdot) \in L^{1}\left(\mathbb{R}^{+}\right) \cap L^{2}\left(\mathbb{R}^{+}\right)$and also $\left(\mathbf{G}(\cdot)-\mathbf{G}_{\infty}\right) \in L^{1}\left(\mathbb{R}^{+}\right) \cap L^{2}\left(\mathbb{R}^{+}\right)$. The relaxation function:

$$
\mathbf{G}(s)=\mathbf{G}_{0}+\int_{0}^{s} \mathbf{G}^{\prime}(t) d s
$$


is well-defined. We have $\mathbf{G}_{0}=\mathbf{G}(0)$. Also, $\mathbf{G}_{\infty}=\mathbf{G}(\infty)=\lim _{s \rightarrow \infty}\left(\mathbf{G}_{0}+\int_{0}^{s} \mathbf{G}^{\prime}(s) d s\right)$ is positive and well-defined as a consequence of the fading memory property. Thermodynamics implies the symmetry of $\mathbf{G}_{0}$ and $\mathbf{G}_{\infty}[7]$, but not the symmetry of $\mathbf{G}(s), s \in \mathbb{R}^{++}$. However, in the following, we assume that $\mathbf{G}(s)$ is a fourth-order symmetric tensor.

For any $f \in L^{2}(\mathbb{R})$, we denote its Fourier transform by

$$
\begin{aligned}
f_{F}(\omega) & =\int_{-\infty}^{\infty} f(\xi) e^{-i \omega \xi} d \xi \\
& =f_{+}(\omega)+f_{-}(\omega) ; \\
f_{+}(\omega)=\int_{0}^{\infty} f(\xi) e^{-i \omega \xi} d \xi ; & f_{-}(\omega)=\int_{-\infty}^{0} f(\xi) e^{-i \omega \xi} d \xi .
\end{aligned}
$$

Only real-valued functions will be considered so that

$$
\bar{f}_{F}(\omega)=f_{F}(-\omega),
$$

where the bar denotes the complex conjugate. Functions defined on $\mathbb{R}^{+}$are identified with functions on $\mathbb{R}$ that vanish identically on $\mathbb{R}^{--}$. For such functions, $f_{F}=f_{c}-i f_{s}$, where $f_{c}, f_{s}$ are respectively the Fourier cosine and sine transforms.

In this notation,

$$
\mathbf{G}_{F}^{\prime}(\omega)=\mathbf{G}_{c}^{\prime}(\omega)-i \mathbf{G}_{s}^{\prime}(\omega)
$$

It is a consequence of the Second Law that [13]

$$
\mathbf{G}_{s}^{\prime}(\omega)<0, \forall \omega \in \mathbb{R}^{++} .
$$

The integrated form of the sine inversion formula gives that:

$$
\mathbf{G}(\xi)-\mathbf{G}_{0}=\frac{2}{\pi} \int_{0}^{\infty} \frac{1-\cos \omega \xi}{\omega} \mathbf{G}_{s}^{\prime}(\omega) d \omega<0 .
$$

We assume that $\mathbf{G}^{\prime}(0)$ exists, is bounded and nonzero. Then from (5.3) we have $\mathbf{G}^{\prime}(0)<0$.

When $\xi \rightarrow \infty$ in (5.3) we also obtain

$$
\mathbf{G}_{\infty}-\mathbf{G}_{0}=\frac{2}{\pi} \int_{0}^{\infty} \frac{\mathbf{G}_{s}^{\prime}(\omega)}{\omega} d \omega<0,
$$

which yields $\mathbf{G}_{s}^{\prime} / \omega \in L^{1}\left(\mathbb{R}^{+}\right)$. Incidentally, from (5.4) we have

$$
\mathbf{G}_{0}>\mathbf{G}_{\infty} \text {. }
$$

Since $\mathbf{G}_{\infty}$ is positive definite, it follows that $\mathbf{G}_{0}$ also has this property.

If $f, g \in L^{2}(\mathbb{R})$, Plancherel's theorem for the Fourier transform gives

$$
\int_{-\infty}^{\infty} g(\xi) f(\xi) d \xi=\frac{1}{2 \pi} \int_{-\infty}^{\infty} g_{F}(\omega) \bar{f}_{F}(\omega) d \omega .
$$

As a consequence of $\mathbf{G}^{\prime} \in L^{2}\left(\mathbb{R}^{+}\right)$and of Plancherel's theorem, the constitutive equation (5.1) can be written as [3]

$$
\mathbf{T}(t)=\mathbf{G}_{0} \mathbf{E}(t)+\frac{2}{\pi} \int_{0}^{\infty} \mathbf{G}_{s}^{\prime}(\omega) \mathbf{E}_{s}^{t}(\omega) d \omega
$$


for any $\mathbf{E}^{t} \in L^{2}\left(\mathbb{R}^{+}\right)$. Now it is important to generalize (5.6) so that it holds for all $\mathbf{E}^{t} \in \mathcal{E}$, where $\mathcal{E}$ is the set of histories $\mathbf{E}^{t}$ such that

$$
\left|\int_{0}^{\infty} \mathbf{G}^{\prime}(s) \mathbf{E}^{t}(s) d s\right|<\infty
$$

for a given $\mathbf{G}^{\prime} \in L^{2}\left(\mathbb{R}^{+}\right) \cap L^{1}\left(\mathbb{R}^{+}\right)$. If we denote by $\mathcal{F}$ the vector space defined by

$$
\mathcal{F}=\left\{\mathbf{F}: \mathbb{R}^{+} \rightarrow \operatorname{Lin}(\operatorname{Sym}, \operatorname{Sym}) ; \mathbf{F}=\alpha \mathbf{G}^{\prime}+\mathbf{f}, \forall \mathbf{f} \in \mathbf{C}_{0}^{\infty}\left(\mathbb{R}^{+}\right)\right\},
$$

then $\mathcal{E}=\mathcal{F}^{\prime}$, where $\mathcal{F}^{\prime}$ is the space of all linear continuous functionals on $\mathcal{F}$. Thus the elements of $\mathcal{E}$ have a Fourier transform in a distributional sense. Then the relation (5.6) can be carried over into the set $\mathcal{E}$.

6. Maximum recoverable work. We denote by $\mathbf{G}(|s|)$ the extension of $\mathbf{G}(s)$ to an even function on $\mathbb{R}$. Also, we suppose that any process $P \in \Pi$ is defined over $\mathbb{R}^{+}$by means of the trivial extension

$$
P(t)= \begin{cases}P(t), & t \in\left[0, d_{p}\right), \\ 0, & t \in\left[d_{p}, \infty\right) .\end{cases}
$$

Now let us evaluate the work $W\left(\sigma_{0}, P\right)$, where $\sigma_{0}=\mathbf{E}^{0}$ is the history at $t=0$. We consider states at $t=0$ in this section and the next, for convenience. There is no loss of generality in doing so. In later sections, the discussion is based on states at time $t$, not necessarily zero. Also, $P \in \Pi$ is a process such that $P(t)=\dot{\mathbf{E}}(t), t \in\left[0, d_{p}\right)$. We have $\mathbf{E}^{t}=\hat{\rho}\left(\mathbf{E}^{0}, P_{t}\right)$ and the stress is given by

$$
\mathbf{T}\left(\mathbf{E}^{t}\right)=\mathbf{G}_{0} \mathbf{E}(t)+\int_{0}^{t} \mathbf{G}^{\prime}(s) \mathbf{E}^{t}(s) d s-\mathbf{I}_{0}\left(t, \mathbf{E}^{0}\right),
$$

where

$$
\mathbf{I}_{0}\left(t, \mathbf{E}^{0}\right)=-\int_{0}^{\infty} \mathbf{G}^{\prime}(t+\tau) \mathbf{E}^{0}(\tau) d \tau, \quad t \geq 0 .
$$

Moreover, from (6.1) it follows that there exists the limit $\mathbf{E}(\infty)=\lim _{t \rightarrow+\infty} \mathbf{E}(t)$. We have

$$
\begin{gathered}
W(\sigma, P)=\int_{0}^{\infty}\left(\mathbf{G}_{0} \mathbf{E}(t)+\int_{0}^{t} \mathbf{G}^{\prime}(s) \mathbf{E}^{t}(s) d s\right) \cdot \dot{\mathbf{E}}(t) d t \\
-\int_{0}^{\infty} \mathbf{I}_{0}\left(t, \mathbf{E}^{0}\right) \cdot \dot{\mathbf{E}}(t) d t=\int_{0}^{\infty}\left(\mathbf{G}_{0} \mathbf{E}(t) \cdot \dot{\mathbf{E}}(t)\right. \\
\left.+\int_{0}^{\infty}\left\{\left.\mathbf{G}(s) \mathbf{E}(t-s)\right|_{0} ^{t}+\int_{0}^{t} \mathbf{G}(s) \dot{\mathbf{E}}(t-s) d s\right)\right\} \cdot \dot{\mathbf{E}}(t) d t \\
\quad-\int_{0}^{\infty} \mathbf{I}_{0}\left(t, \mathbf{E}^{0}\right) \cdot \dot{\mathbf{E}}(t) d t \\
=\frac{1}{2} \int_{0}^{\infty} \int_{0}^{\infty} \mathbf{G}(|t-\tau|) \dot{\mathbf{E}}(\tau) \cdot \dot{\mathbf{E}}(t) d \tau d t-\int_{0}^{\infty} \tilde{\mathbf{I}}_{0}\left(t, \mathbf{E}^{0}\right) \cdot \dot{\mathbf{E}}(t) d t
\end{gathered}
$$


where

$$
\tilde{\mathbf{I}}_{0}\left(t, \mathbf{E}^{0}\right)=-\mathbf{G}(t) \mathbf{E}(0)+\mathbf{I}_{0}\left(t, \mathbf{E}^{0}\right) .
$$

In order to obtain the maximum recoverable work from state $\sigma_{0}=\mathbf{E}^{0}$, we consider the maximum of $-W\left(\sigma_{0}, P\right)$ with respect to the set of functions given by

$$
\mathbf{E}(t)=\mathbf{E}^{(m)}(t)+\varepsilon \mathbf{e}(t), \quad t \in \mathbb{R}^{+},
$$

where $\varepsilon$ is a real parameter and $\mathbf{e}$ is an arbitrary smooth function such that $\mathbf{e}(0)=0$. If $\mathbf{E}^{(m)}$ is the process from which we obtain the maximum recoverable work, then

$$
\begin{aligned}
\frac{d}{d \varepsilon}[-W(\sigma, P)]_{\varepsilon=0}= & -\int_{0}^{\infty} \int_{0}^{\infty} \mathbf{G}(|t-\tau|) \dot{\mathbf{E}}^{(m)}(t) \cdot \dot{\mathbf{e}}(\tau) d \tau d t \\
& +\int_{0}^{\infty} \tilde{\mathbf{I}}_{0}\left(t, \mathbf{E}^{0}\right) \cdot \dot{\mathbf{e}}(t) d t=0
\end{aligned}
$$

Since $\dot{\mathbf{e}}(t)$ is arbitrary, we obtain for $t \in \mathbb{R}^{+}$,

$$
\int_{0}^{\infty} \mathbf{G}(|t-\tau|) \dot{\mathbf{E}}^{(m)}(\tau) d \tau=\tilde{\mathbf{I}}_{0}\left(t, \mathbf{E}^{0}\right)
$$

Equation (6.5) is an integral equation of the Wiener-Hopf type, the solution of which gives the process $\dot{\mathbf{E}}^{(m)}$, which yields the maximum recoverable work. We have from Theorem 4.1 and relations (6.3), (6.5) that

$$
\psi_{m}\left(\mathbf{E}^{0}\right)=\frac{1}{2} \int_{0}^{\infty} \int_{0}^{\infty} \mathbf{G}(|t-\tau|) \dot{\mathbf{E}}^{(m)}(\tau) \cdot \dot{\mathbf{E}}^{(m)}(t) d \tau d t
$$

where $\dot{\mathbf{E}}^{(\mathbf{m})}$ is now the solution of the Wiener-Hopf equation (6.5).

It is important to prove the existence and uniqueness of the solution of (6.5). Let us assume that the kernel $\mathbf{G}(|t|)$ is a positive operator. We denote by

$$
\mathcal{G}=\left\{\dot{\mathbf{E}}:[0, \infty) \rightarrow \operatorname{Sym} ; \int_{0}^{\infty} \int_{0}^{\infty} \mathbf{G}(|t-\tau|) \dot{\mathbf{E}}(t) \cdot \dot{\mathbf{E}}(\tau) d \tau d t<\infty\right\}
$$

We can introduce an inner product on $\mathcal{G}$ by

$$
\left(\dot{\mathbf{E}}_{1}, \dot{\mathbf{E}}_{2}\right)=\int_{0}^{\infty} \int_{0}^{\infty} \mathbf{G}(|t-\tau|) \dot{\mathbf{E}}_{1}(\tau) \cdot \dot{\mathbf{E}}_{2}(t) d \tau d t
$$

which makes $\mathcal{G}$ a Hilbert space. The norm on $\mathcal{G}$ is given by

$$
\begin{aligned}
\|\dot{\mathbf{E}}\|^{2} & =\int_{0}^{\infty} \int_{0}^{\infty} \mathbf{G}(|t-\tau|) \dot{\mathbf{E}}(t) \cdot \dot{\mathbf{E}}(\tau) d \tau d t \\
& =\mathbf{G}_{\infty}\left|\int_{0}^{\infty} \dot{\mathbf{E}}(\tau) d \tau\right|^{2}+\int_{0}^{\infty} \int_{0}^{\infty} \check{\mathbf{G}}(|t-\tau|) \dot{\mathbf{E}}(t) \cdot \dot{\mathbf{E}}(\tau) d \tau d t
\end{aligned}
$$

where $\check{\mathbf{G}}(s)=\mathbf{G}(s)-\mathbf{G}_{\infty} \in L^{1}\left(\mathbb{R}^{+}\right)$as assumed earlier. Therefore, the processes $\dot{\mathbf{E}}$ are such that

$$
\begin{aligned}
\int_{0}^{\infty} \dot{\mathbf{E}}(t) d t=\mathbf{E}(\infty)-\mathbf{E}(0) & <\infty \\
\int_{0}^{\infty} \int_{0}^{\infty} \check{\mathbf{G}}(|t-\tau|) \dot{\mathbf{E}}(t) \cdot \dot{\mathbf{E}}(\tau) d \tau d t & <\infty .
\end{aligned}
$$


The first relation essentially states that $\mathbf{E}(\infty)$ is finite, which, as noted earlier, follows from (6.1).

If $\check{\mathbf{G}}$ is given by an exponential function

$$
\check{\mathbf{G}}(s)=A e^{-\alpha s} \mathbf{I},
$$

where $\mathbf{I}$ is the unit operator in $\operatorname{Lin}(\mathrm{Sym})$ and $A$ is a scalar constant, then the space $\mathcal{G}$ is well-defined. In fact, we have, extending the integrals to $\mathbb{R}$ and using the notation of $(5.2)$,

$$
\int_{0}^{\infty} \int_{0}^{\infty} \check{\mathbf{G}}(|t-\tau|) \dot{\mathbf{E}}(t) \cdot \dot{\mathbf{E}}(\tau) d \tau d t=\frac{A}{\pi} \int_{0}^{\infty} \frac{\alpha}{\alpha^{2}+\omega^{2}}\left|\dot{\mathbf{E}}_{F}(\omega)\right|^{2} d \omega
$$

so that $\dot{\mathbf{E}} \in H^{-1}\left(\mathbb{R}^{+}\right)$, the dual of the Sobolev space $H^{1}\left(\mathbb{R}^{+}\right)$. In general, we can affirm that $\mathcal{G} \supset L^{1}\left(\mathbb{R}^{+}\right) \cap L^{2}\left(\mathbb{R}^{+}\right)$.

REMARK 6.1. By means of the norm of $\mathcal{G}$, it is possible to provide the set of the processes $\Pi$ with a topology. In particular, the closure of $\Pi$ using the norm (6.7) is the Hilbert space $\mathcal{G}$.

Equation (6.5) can be written as

$$
\mathcal{A} \dot{\mathbf{E}}=\mathbf{I}_{0},
$$

where $\mathcal{A}$ is an operator from $\mathcal{G}$ to its dual $\mathcal{G}^{\prime}$. It is bounded and coercive. Then, from the Lax-Milgram theorem, we have the following result:

Theorem 6.1. For any $\tilde{\mathbf{I}}_{0} \in \mathcal{G}^{\prime}$, the equation (6.5) has a unique solution $\dot{\mathbf{E}} \in \mathcal{G}$ such that

$$
\|\dot{\mathbf{E}}\|_{\mathcal{G}} \leq K\left\|\tilde{\mathbf{I}}_{0}\right\|_{\mathcal{G}^{\prime}}
$$

In other words, there exists an isomorphism between $\mathcal{G}$ and $\mathcal{G}^{\prime}$.

Proposition 6.2. Two histories $\mathbf{E}_{1}^{0}, \mathbf{E}_{2}^{0}$ correspond to two equivalent states if and only if

$$
\tilde{\mathbf{I}}_{0}\left(t, \mathbf{E}_{1}^{0}\right)=\tilde{\mathbf{I}}_{0}\left(t, \mathbf{E}_{2}^{0}\right) \quad \forall t \in \mathbb{R}^{+}
$$

Proof. If (6.8) holds for any $t \in \mathbb{R}^{+}$, then for $t \rightarrow \infty$ we have

$$
\mathbf{E}_{1}^{0}(0)=\mathbf{E}_{2}^{0}(0) \text {. }
$$

Equations (6.8) and (6.9) imply equivalence according to Definition 3.2. The converse is trivial.

REMARK 6.2. Proposition 6.2 yields a bijective map between $\mathcal{G}^{\prime}$ and the set $\Sigma_{R}$. In other words, it is possible to identify any class of equivalent histories with a function $\tilde{\mathbf{I}}_{0}$.

This result allows us to represent the minimum free energy as a function defined on the set $\Sigma_{R}$ of the equivalent histories, which will be done explicitly for a particular class of materials in Sec. 11 .

Let us now consider the implications of Theorem 4.2 and, in particular, Corollary 4.3. Motivated by the latter result and the fact, stated formally in Sec. 8, that the elastic 
free energy is less than or equal to any viscoelastic free energy, we take $\phi_{M}$ to be $\phi(\infty)$ where $\phi(t)$ is the elastic free energy corresponding to the strain $\mathbf{E}(t)$, given by

$$
\phi(t)=\frac{1}{2} \mathbf{G}_{\infty} \mathbf{E}(t) \cdot \mathbf{E}(t) .
$$

This choice will be justified more clearly later. If we seek the history that maximizes the functional

$$
\begin{aligned}
& W(\sigma, P)-\phi(\infty) \\
& \quad=\frac{1}{2} \int_{0}^{\infty} \int_{0}^{\infty} \mathbf{G}(|t-\tau|) \dot{\mathbf{E}}(\tau) \cdot \dot{\mathbf{E}}(t) d \tau d t-\int_{0}^{\infty} \tilde{\mathbf{I}}_{0}\left(t, \mathbf{E}^{0}\right) \cdot \dot{\mathbf{E}}(t) d t-\phi(\infty),
\end{aligned}
$$

where $\tilde{\mathbf{I}}_{0}\left(t, \mathbf{E}^{0}\right)$ is defined by (6.4) then, instead of (6.5), we obtain

$$
\int_{0}^{\infty} \mathbf{G}(|t-\tau|) \dot{\mathbf{E}}^{(m)}(\tau) d \tau=\tilde{\mathbf{I}}_{0}\left(t, \mathbf{E}^{0}\right)+\mathbf{G}_{\infty} \mathbf{E}^{(m)}(\infty)
$$

noting that $\mathbf{e}(\infty)=\int_{0}^{\infty} \dot{\mathbf{e}}(t) d t$.

Let us express the solution of (6.5), namely $\dot{\mathbf{E}}^{(m)}$, as the sum

$$
\dot{\mathbf{E}}^{(m)}=\dot{\mathbf{E}}^{\left(m_{1}\right)}+\dot{\mathbf{E}}^{\left(m_{2}\right)},
$$

where $\dot{\mathbf{E}}^{\left(m_{1}\right)}$ is the solution of $(6.12)$ and $\dot{\mathbf{E}}^{\left(m_{2}\right)}$ satisfies the equation obtained by subtracting (6.12) from (6.5), namely

$$
\begin{aligned}
\int_{0}^{\infty} \mathbf{G}(|t-\tau|) \dot{\mathbf{E}}^{\left(m_{2}\right)}(\tau) d \tau & =-\mathbf{G}_{\infty} \mathbf{E}^{\left(m_{1}\right)}(\infty) \\
& =-\mathbf{G}_{\infty}\left(\mathbf{E}^{\left(m_{1}\right)}(0)+\int_{0}^{\infty} \dot{\mathbf{E}}^{\left(m_{1}\right)}(\tau) d \tau\right)
\end{aligned}
$$

As a consequence of Theorem 6.1, equation (6.14) has a unique solution $\dot{\mathbf{E}}^{\left(m_{2}\right)} \in \mathcal{G}$, which we denote formally by

$$
\dot{\mathbf{E}}^{\left(m_{2}\right)}(t)=-\mathbf{E}^{\left(m_{1}\right)}(\infty) \delta(t-\infty) .
$$

This function can be obtained as the limit of the sequence

$$
\dot{\mathbf{E}}_{n}^{\left(m_{2}\right)}(t)=\left\{\begin{array}{cc}
0, & t<n, \\
-\frac{\mathbf{E}^{\left(m_{1}\right)}(\infty)}{n}, & n<t<2 n, \\
0, & t>2 n .
\end{array}\right.
$$

Note that (6.13) corresponds to continuations given formally by

$$
\mathbf{E}^{(m)}(t)=\mathbf{E}^{\left(m_{1}\right)}(t)-\mathbf{E}^{\left(m_{1}\right)}(\infty) H(t-\infty),
$$

where $H(s)$ is the Heaviside step-function.

REMARK 6.3. As a consequence of Theorem 4.2, the maximum recoverable work, which we obtain using the functional $W(\sigma, P)$ or $\left(W(\sigma, P)-\phi_{M}(\hat{\rho}(\sigma, P))\right)$ is the same, but the processes from which we obtain this maximum recoverable work are different. If we use $W(\sigma, P)$, the optimal process is $\dot{\mathbf{E}}^{(m)}$, which satisfies (6.5); while if we use $\left(W(\sigma, P)-\phi_{M}(\hat{\rho}(\sigma, P))\right)$, the optimal process is $\dot{\mathbf{E}}^{\left(m_{1}\right)}$, which satisfies $(6.12)$. 
For later use, we note that $(6.12)$ can be written in the form

$$
\begin{aligned}
& \int_{-\infty}^{\infty} \frac{\partial}{\partial t} \mathbf{G}(|t+\tau|) \mathbf{E}^{0}(\tau) d \tau=0, \quad t \in \mathbb{R}^{+}, \\
& \mathbf{E}^{0}(\tau)=\mathbf{E}^{\left(m_{1}\right)}(-\tau), \quad \tau \in \mathbb{R}^{--},
\end{aligned}
$$

while on $\mathbb{R}^{+}$, the quantity $\mathbf{E}^{()}$is the given history. We have from Theorem 4.2 and Corollary $4.3,(6.11)$ and $(6.12)$,

$$
\begin{gathered}
\psi_{m}\left(\mathbf{E}^{0}\right)=\frac{1}{2} \int_{0}^{\infty} \int_{0}^{\infty} \mathbf{G}(|t-\tau|) \dot{\mathbf{E}}^{\left(m_{1}\right)}(\tau) \cdot \dot{\mathbf{E}}^{\left(m_{1}\right)}(t) d \tau d t-\phi(\infty) \\
+\mathbf{G}_{\infty} \mathbf{E}(\infty) \mathbf{E}(0)
\end{gathered}
$$

where $\dot{\mathbf{E}}^{\left(m_{1}\right)}$ is now the solution of the equation (6.12). Carrying out partial integrations and using (6.16), we find that

$$
\begin{aligned}
\psi_{m}\left(\mathbf{E}^{0}\right) & =S(0)+\frac{1}{2} \int_{0}^{\infty} \int_{0}^{\infty} \frac{\partial^{2}}{\partial t \partial \tau} \mathbf{G}(|t-\tau|) \mathbf{E}^{\left(m_{1}\right)}(\tau) \cdot \mathbf{E}^{\left(m_{1}\right)}(t) d \tau d t, \\
S(t) & =\mathbf{T}(t) \cdot \mathbf{E}(t)-\frac{1}{2} \mathbf{G}_{0} \mathbf{E}(t) \cdot \mathbf{E}(t) .
\end{aligned}
$$

The form (6.17) is shown in Sec. 8 (Proposition 8.1) to have the characteristic properties of a free energy, one of which eliminates the freedom of an additive constant. This justifies the inclusion of $\phi(\infty)$ in $(6.11)$.

7. Minimum free energy. We begin with some preliminary results and definitions. Following [4], we will be considering frequency space quantities, defined by analytic continuation from integral definitions, as functions on the complex $\omega$-plane, denoted by $\Omega$, where

$$
\begin{aligned}
\Omega^{+} & =\left\{\omega \in \Omega \mid \Im \omega \in \mathbb{R}^{+}\right\}, \\
\Omega^{(+)} & =\left\{\omega \in \Omega \mid \Im \omega \in \mathbb{R}^{++}\right\} .
\end{aligned}
$$

Similarly, $\Omega^{-}$and $\Omega^{(-)}$are the lower half-planes including and excluding the real axis, respectively.

The quantities $f_{ \pm}$, defined by (5.2), are analytic in $\Omega^{(\mp)}$ respectively. In fact, the relation $f_{F}(\omega)=f_{+}(\omega)+f_{-}(\omega)$ is equivalent to the Plemelj formula [14] which will be used later.

The function $\mathbf{G}_{F}^{\prime}$ is analytic on $\Omega^{(-)}$. 'This follows from its integral definition over $\mathbb{R}^{+}$ and may be viewed as a consequence of Causality [15]. It will be assumed further that $\mathbf{G}_{F}^{\prime}$ is analytic on $\mathbb{R}$ and thus on $\Omega^{-}$. It is defined by analytic continuation in regions of $\Omega^{+}$where the Fourier integral does not converge.

The quantity $\mathbf{G}_{s}^{\prime}$ has singularities in both $\Omega^{(+)}$and $\Omega^{(-)}$that are mirror images of each other. It goes to zero at the origin and must also be analytic there. Thus, it vanishes as $\omega^{n}$ where the integer $n \geq 1$. It is assumed that $n=1$.

A quantity central to our considerations is defined by

$$
\mathbf{H}(\omega)=-\omega \mathbf{G}_{s}^{\prime}(\omega) .
$$


It is a positive, even function of the frequency. The relation

$$
i \lim _{\omega \rightarrow \infty} \omega \mathbf{G}_{F}^{\prime}(\omega)=\lim _{\omega \rightarrow \infty} \omega \mathbf{G}_{s}^{\prime}(\omega)=\mathbf{G}^{\prime}(0)
$$

yields

$$
\mathbf{G}^{\prime}(0)=-\mathbf{H}(\infty)
$$

If $\mathbf{G}(s), s \in \mathbb{R}^{+}$, is extended to the even function $\mathbf{G}(|s|)$ on $\mathbb{R}$, then $\mathbf{G}^{\prime}(|s|)$ is an odd function with Fourier transform given by

$$
\mathbf{G}_{F}^{\prime}(\omega)=-2 i \mathbf{G}_{s}^{\prime}(\omega) \text {. }
$$

We will be using the Fourier transforms of the strain history and continuation, denoted by $\mathbf{E}_{+}^{t}(\omega)$ and $\mathbf{E}_{-}^{t}(\omega)$ respectively. It is necessary to include cases where these do not belong to $L^{2}$. Consider the case where $\mathbf{E}(-\infty) \neq 0$ but with $\mathbf{E}_{d+}^{t} \in L^{2}\left(\mathbb{R}^{+}\right)$defined by

$$
\mathbf{E}_{d+}^{t}(s)=\mathbf{E}^{t}(s)-\mathbf{E}(-\infty)
$$

In this case, we write

$$
\begin{aligned}
\mathbf{E}_{+}^{t}(\omega) & =\int_{0}^{\infty} e^{-i \omega s} \mathbf{E}^{t}(s) d s \\
& =\int_{0}^{\infty} e^{-i \omega s} \mathbf{E}_{d+}^{t}(s) d s+\mathbf{E}(-\infty) \int_{0}^{\infty} e^{-i \omega s} d s \\
& =\mathbf{E}_{d+}^{t}(\omega)+\frac{\mathbf{E}(-\infty)}{i \omega^{-}} ; \quad \omega^{-}=\lim _{\alpha \rightarrow 0}(\omega-i \alpha),
\end{aligned}
$$

where the limit is taken after any integrations in frequency space are carried out. This well-known device for handling such functions-effectively as a limit of $L^{2}$ functions-is in fact, in the present context, largely redundant since $\mathbf{E}_{+}^{t}(\omega)$ will generally multiply functions such as $\mathbf{G}_{s}^{\prime}(\omega)$ that vanish at $\omega=0$ in such a way as to cancel the pole. This is a particular manifestation of the remarks at the end of Sec. 5 .

Also, we define $\mathbf{E}_{-}^{t}(\omega)$ to be

$$
\begin{aligned}
\mathbf{E}_{-}^{t}(\omega) & =\int_{-\infty}^{0} e^{-i \omega s} \mathbf{E}^{t}(s) d s \\
& =\mathbf{E}_{d-}^{t}(\omega)-\frac{\mathbf{E}(\infty)}{i \omega^{+}}, \omega^{+}=\lim _{\alpha \rightarrow 0}(\omega+i \alpha), \\
\mathbf{E}_{d-}^{t}(\omega) & =\mathbf{E}_{-}^{t}(\omega)-\mathbf{E}(\infty),
\end{aligned}
$$

where we allow the possibility that $\mathbf{E}(\infty) \neq 0$.

The quantity $\mathbf{E}_{+}^{t}$ is analytic on $\Omega^{(-)}$and $\mathbf{E}_{-}^{t}$ is analytic on $\Omega^{(+)}$. Both are assumed to be analytic on $\mathbb{R}$. Poles that may exist near the origin are moved slightly off the real axis as prescribed in (7.6) and (7.7). It is further assumed that they are analytic at infinity, so that if $\mathbf{E}^{t}(0)$ is finite, which is of course assumed, $\mathbf{E}_{ \pm}^{t}$ go to zero at large $\omega$ as $\omega^{-1}$ in all directions. We will require the derivative of $\mathbf{E}_{+}^{t}$ with respect to $t$. Assuming that the strain history has a derivative that is continuous and belongs to $L^{1}\left(\mathbb{R}^{+}\right)$, then

$$
\frac{d}{d t} \mathbf{E}_{+}^{t}(\omega)=-i \omega \mathbf{E}_{+}^{t}(\omega)+\mathbf{E}(t)
$$


Observe that from (7.6),

$$
i \lim _{\omega \rightarrow 0} \omega \mathbf{E}_{+}^{t}(\omega)=\lim _{\omega \rightarrow 0} \omega \mathbf{E}_{s}^{t}(\omega)=\mathbf{E}(-\infty) .
$$

Also (see (7.3)),

$$
i \lim _{\omega \rightarrow \infty} \omega \mathbf{E}_{+}^{t}(\omega)=\lim _{\omega \rightarrow \infty} \omega \mathbf{E}_{s}^{t}(\omega)=\mathbf{E}(t) .
$$

We can write (5.6) in the form

$$
\begin{aligned}
\mathbf{T}(t) & =\mathbf{G}_{0} \mathbf{E}(t)+\frac{1}{2 \pi i} \int_{-\infty}^{\infty} \frac{\mathbf{H}(\omega)}{\omega}\left(\mathbf{E}_{+}^{t}(\omega)-\mathbf{E}_{+}^{t}(-\omega)\right) d \omega \\
& =\mathbf{G}_{0} \mathbf{E}(t)+\frac{1}{i \pi} \int_{-\infty}^{\infty} \frac{\mathbf{H}(\omega)}{\omega} \mathbf{E}_{+}^{t}(\omega) d \omega,
\end{aligned}
$$

where the oddness of $\mathbf{H}(\omega) / \omega$ has been used in writing both forms.

Let us now solve the Wiener-Hopf equation (6.12) or (6.16). Replacing the parameter $t$ by $-t$, we write the latter form as

$$
\begin{aligned}
\int_{-\infty}^{\infty} \frac{\partial}{\partial t} \mathbf{G}(|t-\tau|) \mathbf{E}^{0}(\tau) d \tau & =\mathbf{R}(t) \\
\mathbf{R}(t) & =0, \quad t \in \mathbb{R}^{-} .
\end{aligned}
$$

This relation defines $\mathbf{R}$ on $\mathbb{R}^{+}$. Taking Fourier transforms and multiplying across by $\omega$, we obtain, with the aid of (7.5) and (7.2),

$$
2 i \mathbf{H}(\omega)\left(\mathbf{E}_{+}^{0}(\omega)+\mathbf{E}^{(m)}(\omega)\right)=\omega \mathbf{R}_{+}(\omega)
$$

where $\mathbf{E}^{(m)}(\omega)$ is the Fourier transform of $\mathbf{E}^{0}(\tau)$ defined by (6.16) on $\mathbb{R}^{--}$and is the quantity we wish to determine. Also, $\mathbf{R}_{+}(\omega)$ is analytic on $\Omega^{(-)}$and by assumption also on $\mathbb{R}$.

Now [5], the tensor $\mathbf{H}$ (which is isomorphic to a matrix in $\mathbb{R}_{6} \times \mathbb{R}_{6}$ ) can be factorized as follows: $\mathbf{H}(\omega)=\mathbf{H}_{+}(\omega) \mathbf{H}_{-}(\omega)$, where $\mathbf{H}_{ \pm}$is analytic, with no zeros in its determinant, on $\Omega^{\mp}$. We multiply $(7.13)$ by $\left[\mathbf{H}_{+}(\omega)\right]^{-1}$ to obtain

$$
\mathbf{H}_{-}(\omega)\left(\mathbf{E}_{+}^{0}(\omega)+\mathbf{E}^{(m)}(\omega)\right)=\frac{\omega}{2 i}\left[\mathbf{H}_{+}(\omega)\right]^{-1} \mathbf{R}_{+}(\omega)
$$

Now, with the aid of the Plemelj formulae [14], we write

$$
\begin{aligned}
\mathbf{Q}(\omega):=\mathbf{H}_{-}(\omega) \mathbf{E}_{+}^{0}(\omega) & =\mathbf{q}_{-}(\omega)-\mathbf{q}_{+}(\omega), \\
\mathbf{q}_{ \pm}(\omega) & =\lim _{z \rightarrow \omega \mp} \mathbf{q}(z), \\
\mathbf{q}(z) & =\frac{1}{2 \pi i} \int_{-\infty}^{\infty} \frac{\mathbf{Q}\left(\omega^{\prime}\right)}{\omega^{\prime}-z} d \omega^{\prime},
\end{aligned}
$$

where $\mathbf{q}_{-}$is analytic on $\Omega^{(+)}$and $\mathbf{q}_{+}$is analytic on $\Omega^{(-)}$. In fact, they are given by $\mathbf{q}(z)$ for $z \in \Omega^{(+)}$and $\Omega^{(-)}$respectively. With earlier assumptions giving that $\mathbf{H}_{-}(\omega) \mathbf{E}_{+}^{0}(\omega)$ is analytic on $\mathbb{R}$, it can be deduced that $\mathbf{q}_{ \pm}$are analytic on $\mathbb{R}[4]$. In $\Omega^{(-)}$, $\mathbf{q}_{-}$is defined by analytic continuation from $\Omega^{+}$, while $\mathbf{q}_{+}$is correspondingly defined in $\Omega^{(+)}$. 
Substituting (7.15) into (7.14) we obtain

$$
\mathbf{K}(\omega)=\mathbf{q}_{-}(\omega)+\mathbf{H}_{-}(\omega) \mathbf{E}^{(m)}(\omega)=\mathbf{q}_{+}(\omega)+\frac{\omega}{2 i}\left[\mathbf{H}_{+}(\omega)\right]^{-1} \mathbf{R}_{+}(\omega)
$$

The function $\mathbf{K}(\omega)$ is analytic on $\Omega^{-}$by virtue of the first relation and analytic on $\Omega^{+}$by virtue of the second. It is therefore analytic over the entire complex plane. By Liouville's theorem it must be a polynomial. However, for $|\omega| \rightarrow \infty, \mathbf{K}(\omega) \rightarrow 0$ as $1 / \omega$ since $\mathbf{q}_{-}$ and $\mathbf{E}^{(m)}$ have this property. Hence, it must vanish everywhere so that

$$
\mathbf{H}_{-}(\omega) \mathbf{E}^{(m)}(\omega)+\mathbf{q}_{-}(\omega)=0
$$

and the minimum free energy (6.17) may be represented in the form

$$
\begin{aligned}
\psi_{m}\left(\mathbf{E}^{0}\right) & =S(0)+\frac{1}{2 \pi} \int_{-\infty}^{\infty} \mathbf{H}(\omega) \mathbf{E}^{(m)}(\omega) \cdot \overline{\mathbf{E}}^{(m)}(\omega) d \omega \\
& =S(0)+\frac{1}{2 \pi} \int_{-\infty}^{\infty} \mathbf{q}_{-}(\omega) \cdot \overline{\mathbf{q}}_{-}(\omega) d \omega
\end{aligned}
$$

by application of the Convolution theorem, Plancherel's theorem (5.5) and (7.17). The results (7.17) and (7.18) agree with those in [5] obtained by a variational technique.

The above solution can be generalized to the set of histories $\mathcal{E}$ defined by (5.7). We write $(7.12)$ in the form

$$
\begin{aligned}
\int_{-\infty}^{0} \frac{\partial}{\partial t} \mathbf{G}(|t-\tau|) \mathbf{E}^{0}(\tau) d \tau-\mathbf{J}(t) & =\mathbf{R}(t), \quad t \in \mathbb{R} \\
\mathbf{J}(t) & =\mathbf{I}_{0}\left(-t, \mathbf{E}^{0}\right)
\end{aligned}
$$

where $\mathbf{I}_{0}\left(t, \mathbf{E}^{0}\right)$ is the quantity defined by (6.2), defined for $t \in \mathbb{R}$ through the even extension of $\mathbf{G}$. We have $\mathbf{J} \in L^{2}(\mathbb{R})$ by virtue of Definition 2.1 of fading memory, on recalling that $\mathbf{G}(\cdot)-\mathbf{G}_{\infty} \in L^{2}\left(\mathbb{R}^{+}\right)$as assumed earlier. This is easily shown for $L^{2}\left(\mathbb{R}^{-}\right)$, i.e., before extension to $\mathbb{R}$. The extension is achieved by observing that it is essentially a statement about the behaviour of $\mathbf{J}$ for large $|t|$. Denoting its Fourier transform by $\mathbf{J}_{F} \in L^{2}(\mathbb{R})$, we obtain, instead of (7.13),

$$
2 i \mathbf{H}(\omega) \mathbf{E}^{(m)}(\omega)-\omega \mathbf{J}_{F}(\omega)=\omega \mathbf{R}_{+}(\omega)
$$

The argument proceeds as outlined above but where $\mathbf{Q}(\omega)$ in (7.15) is now defined by

$$
\mathbf{Q}(\omega)=-\frac{\omega}{2 i}\left[\mathbf{H}_{+}(\omega)\right]^{-1} \mathbf{J}_{F}(\omega)
$$

It is assumed that $\mathbf{J}_{F}$ is analytic on $\mathbb{R}$. The analytic Fourier transform on $\mathbb{R}$ of a function that is continuous at $t=0$ behaves as $\omega^{-2}$ at large frequencies, so that $\mathbf{J}_{F}$ has this property. Thus, $\mathbf{Q}(\omega) \sim \omega^{-1}$ at large $\omega$, as required for the convergence of the integral in (7.15). 


\section{Part B: A NEW VARIATIONAL METHOD.}

8. Certain time-domain relationships. In this first section of Part B, we shall present certain observations and demonstrations, the object of which is to elucidate the content of the developments of later sections in the frequency domain, by pointing to equivalent developments, as far as possible, in the time domain.

Let us consider various forms of the work function:

$$
\begin{aligned}
W(t) & =\phi(-\infty)+\int_{-\infty}^{t} \mathbf{T}(s) \cdot \dot{\mathbf{E}}(s) d s \\
& =S(t)-S(-\infty)+\phi(-\infty)-\int_{-\infty}^{t} \dot{\mathbf{T}}_{1}(s) \cdot \mathbf{E}(s) d s, \\
\mathbf{T}_{1}(s) & =\mathbf{T}(s)-\mathbf{G}_{0} \mathbf{E}(s) .
\end{aligned}
$$

The quantity $\phi(t)$ is the elastic free energy given in (6.10) while $S(t)$ is defined in (6.17). These relations depend on $\mathbf{E}(-\infty)$, which must be assumed to be finite. Note that this is not necessarily the case for strain histories $\mathbf{E}^{t} \in \mathcal{E}$ defined by (5.7). We need not assume however that $\mathbf{E}(-\infty)$ is zero. Using a standard form of (5.1):

$$
\mathbf{T}(t)=\mathbf{G}_{\infty} \mathbf{E}(-\infty)+\int_{-\infty}^{t} \mathbf{G}(t-s) \dot{\mathbf{E}}(s) d s,
$$

one can show that

$$
\begin{aligned}
W(t) & =\phi(-\infty)+g(t)+\frac{1}{2} \int_{-\infty}^{t} \int_{-\infty}^{t} \mathbf{G}(s-u) \dot{\mathbf{E}}(u) \cdot \dot{\mathbf{E}}(s) d u d s, \\
g(t) & =\mathbf{G}_{\infty} \mathbf{E}(-\infty) \cdot(\mathbf{E}(t)-\mathbf{E}(-\infty)),
\end{aligned}
$$

where we understand throughout this section that $\mathbf{G}(s)$ refers to its even extension $\mathbf{G}(|s|), s \in \mathbb{R}$. Partial integrations give that

$$
\begin{aligned}
W(t) & =\phi(t)+\frac{1}{2} \int_{-\infty}^{t} \int_{-\infty}^{t} \mathbf{G}_{12}(s-u)(\mathbf{E}(u)-\mathbf{E}(t)) \cdot(\mathbf{E}(s)-\mathbf{E}(t)) d u d s \\
& =S(t)+\frac{1}{2} \int_{-\infty}^{t} \int_{-\infty}^{t} \mathbf{G}_{12}(s-u) \mathbf{E}(u) \cdot \mathbf{E}(s) d u d s \\
\mathbf{G}_{12}(s-u) & =\frac{\partial^{2}}{\partial u \partial s} \mathbf{G}(s-u) .
\end{aligned}
$$

Note that these forms do not depend on $\mathbf{E}(-\infty)$ and hold even if this quantity is not well-defined. It is the second form of $W(t)$, given by $(8.2)$, that we will use. It may be written as

$$
W(t)=S(t)+\frac{1}{2} \int_{0}^{\infty} \int_{0}^{\infty} \mathbf{G}_{12}(s-u) \mathbf{E}^{t}(u) \cdot \mathbf{E}^{t}(s) d u d s .
$$

Observe that the minimum free energy, given by (6.17), has the same form as (8.3) at $t=0$, where $\mathbf{E}^{0}$ is identified with $\mathbf{E}^{(m)}$. In other words, the history is the time-reversed optimal continuation. This is the situation referred to in Remark 4.4, as becomes clear on noting the form of $W(t)$ given by $(8.1)_{1}$. 
A frequency representation can also be given as in (7.18):

$$
W(t)=S(t)+\frac{1}{2 \pi} \int_{-\infty}^{\infty} \mathbf{H}(\omega) \mathbf{E}_{+}^{t}(\omega) \cdot \overline{\mathbf{E}}_{+}^{t}(\omega) d \omega
$$

where $\mathbf{H}$ is defined by (7.2) and $\mathbf{E}_{+}^{t}$ by (7.6). Note that, in frequency space, the integral term is clearly nonnegative since $\mathbf{H}$ is positive-definite.

We define the scalar product for $\mathbf{E}_{1}, \mathbf{E}_{2}: \mathbb{R} \mapsto$ Sym (differing from, although related to $(6.6)$, if $\mathbf{E}_{1}, \mathbf{E}_{2}$ are zero on $\mathbb{R}^{--}$) by

$$
\begin{aligned}
\left(\mathbf{E}_{1}, \mathbf{E}_{2}\right) & =\frac{1}{2} \int_{-\infty}^{\infty} \int_{-\infty}^{\infty} \mathbf{G}_{12}(s-u) \mathbf{E}_{1}^{t}(u) \cdot \mathbf{E}_{2}^{t}(s) d u d s \\
& =\frac{1}{2 \pi} \int_{-\infty}^{\infty} \mathbf{H}(\omega) \mathbf{E}_{1 F}^{t}(\omega) \cdot \overline{\mathbf{E}}_{2 F}^{t}(\omega) d \omega \\
& =\left(\mathbf{E}_{2}, \mathbf{E}_{1}\right)
\end{aligned}
$$

by virtue of the symmetry of the tensor $\mathbf{G}_{12}$, where

$$
\begin{aligned}
\mathbf{E}_{F}^{t}(\omega) & =\int_{-\infty}^{\infty} e^{-i \omega s} \mathbf{E}^{t}(s) d s \\
& =\mathbf{E}_{+}^{t}(\omega)+\mathbf{E}_{-}^{t}(\omega) .
\end{aligned}
$$

If quantities defined in frequency space are in the brackets, it is understood that the second form of (8.5) is to be used. The norm of $\mathbf{E}$ is defined by

$$
\begin{aligned}
\|\mathbf{E}\|^{2} & =(\mathbf{E}, \mathbf{E}) \geq 0 \\
& =\left\|\mathbf{E}_{F}\right\|^{2}=\left(\mathbf{E}_{F}, \mathbf{E}_{F}\right) .
\end{aligned}
$$

This corresponds to the integral terms in (8.3) and (8.4) if $\mathbf{E}^{t}$ vanishes on $\mathbb{R}^{-}$or, equivalently, if $\mathbf{E}_{-}^{t}(\omega)$ vanishes. Note that, for such a history,

$$
\left(\mathbf{T}(t)-\mathbf{G}_{0} \mathbf{E}(t)\right) \cdot \mathbf{E}_{a}=-2\left(\mathbf{E}_{h}, \mathbf{E}^{t}\right)
$$

for arbitrary $\mathbf{E}_{a} \in \mathrm{Sym}$, where $\mathbf{E}_{h}$ is a constant history equal to $\mathbf{E}_{a}$ and $\left(\mathbf{E}(t), \mathbf{E}^{t}\right)$ is the state resulting in stress $\mathbf{T}(t) .{ }^{4}$ Its Fourier transform (see $(7.6)$ ) is $\mathbf{E}_{a}\left(i \omega^{-}\right)^{-1}$, which yields (7.11).

Let $\mathbf{E}^{t}$ be a given history with zero continuation, i.e., $\mathbf{E}^{t}(s), s \in \mathbb{R}^{--}$vanishes. Also, let $\mathbf{E}(-\infty)=0$. We have $\mathbf{E}^{t}\left(0^{+}\right)=\mathbf{E}(t)$, a specified quantity. Let

$$
\mathbf{E}_{1}^{t}=\mathbf{E}^{t}+\mathbf{E}_{d}^{t}
$$

where in general $\mathbf{E}_{1}^{t}, \mathbf{E}_{d}^{t}$ are not assumed to vanish on $\mathbb{R}^{--}$. The constraint

$$
\int_{-\infty}^{\infty} \mathbf{G}_{2}(s-u) \mathbf{E}_{d}^{t}(u) d u=0, \quad s \in \mathbb{R}^{-},
$$

is imposed, where the subscript 2 indicates differentiation with respect to $u$, giving a discontinuous function. It implies that

$$
\int_{-\infty}^{\infty} \mathbf{G}_{12}(s-u) \mathbf{E}_{d}^{t}(u) d u=0, \quad s \in \mathbb{R}^{-} .
$$

\footnotetext{
${ }^{4}$ The bracket notation for scalar product and state may be distinguished by the fact that, in the latter case, the argument of $\mathbf{E}(t)$ is explicitly included.
} 
In fact, (8.10) also implies (8.9) by virtue of the fact that $\mathbf{G}_{2}$ vanishes at large times. We seek a choice of $\mathbf{E}_{1}^{t}$ that minimizes $\left\|\mathbf{E}_{1}^{t}\right\|^{2}$ under specified variations subject to (8.10). Two cases will be considered, corresponding, as we shall see, to the minimum and maximum free energy. In the frequency domain formulation in Sec. 11, a more general and somewhat different variational method is developed which yields, in addition, a range of intermediate free energies. No equivalent time domain formulation has yet been established, and may in fact not be possible without invoking the time-domain equivalent of factorization in the frequency domain. We will see that the variational process in the frequency domain is implemented after factorization has been carried out.

Let $\mathbf{E}_{1}^{t}=0$ on $\mathbb{R}^{++}$and vary $\mathbf{E}_{d}^{t}$ (or equivalently $\mathbf{E}_{1}^{t}$ ) according to

$$
\mathbf{E}_{d}^{t}(u) \rightarrow \mathbf{E}_{d}^{t}(u)+\varepsilon \mathbf{e}(u), \quad u \in \mathbb{R}^{-}
$$

where $\varepsilon$ is a real parameter and $\mathbf{e}$ is a smooth function. Using a generalization of the Lagrange multiplier technique, we minimize with respect to $\varepsilon$ the quantity

$$
\left(\mathbf{E}_{1}^{t}+\varepsilon \mathbf{e}, \mathbf{E}_{1}^{t}+\varepsilon \mathbf{e}\right)+2\left(\boldsymbol{\Lambda}^{t}, \mathbf{E}_{d}^{t}+\varepsilon \mathbf{e}\right),
$$

where $\Lambda^{t} \in$ Sym and vanishes on $\mathbb{R}^{+}$. The resultant optimizing equations are

$$
\begin{aligned}
\int_{-\infty}^{0} \mathbf{G}_{2}(s-u) \mathbf{E}_{m}^{t}(u) d u & =-\int_{-\infty}^{0} \mathbf{G}_{2}(s-u) \mathbf{\Lambda}^{t}(u) d u, s \in \mathbb{R}^{-} \\
\int_{-\infty}^{0} \mathbf{G}_{2}(s-u) \mathbf{E}_{m}^{t}(u) d u & =\mathbf{f}(s), \quad s \in \mathbb{R}^{-} \\
\mathbf{f}(s) & =\int_{0}^{\infty} \mathbf{G}_{2}(s-u) \mathbf{E}^{t}(u) d u
\end{aligned}
$$

where a differentiation with respect to $s$ has been removed from the first relation, using again the fact that $\mathbf{G}_{2}$ vanishes at large times. The second relation is simply a restatement of the integral condition (8.9) in this case. The quantity $\mathbf{E}_{m}^{t}$ is the optimal choice of $\mathbf{E}_{1}^{t}$.

The first relation yields $\Lambda^{t}=-\mathbf{E}_{m}^{t}$ on $\mathbb{R}^{-}$while the second relation has the same form as (7.12), for $s \in \mathbb{R}^{-}$, which is the equation for the continuation associated with the minimum free energy and which has been solved. The optimal continuation is identified with $\left(-\mathbf{E}_{m}^{t}\right)$. Thus, the optimization is trivial in this case and the real content lies in the constraint (8.9) which is in effect (7.12).

Now consider the case where $\mathbf{E}_{1}^{t}=0$ on $\mathbb{R}^{--}$. The constraint (8.9) is then effectively the same as (6.8) so that the state $\left(\mathbf{E}(t), \mathbf{E}_{m}^{t}\right)$ is equivalent to $\left(\mathbf{E}(t), \mathbf{E}^{t}\right)$. We impose the variation as in (8.11) but now where $\mathbf{e}$ vanishes on $\mathbb{R}^{-}$. The optimizing equations for this case are

$$
\begin{aligned}
\int_{0}^{\infty} \mathbf{G}_{2}(s-u) \mathbf{E}_{m}^{t}(u) d u & =-\int_{-\infty}^{0} \mathbf{G}_{2}(s-u) \mathbf{\Lambda}^{t}(u) d u, s \in \mathbb{R}^{++} \\
& =\mathbf{f}(s), \quad s \in \mathbb{R}^{-}
\end{aligned}
$$


where $\mathbf{f}$ is defined in (8.12). Again a differentiation has been removed. We can write (8.13) in the form

$$
\begin{aligned}
& \int_{-\infty}^{\infty} \mathbf{G}_{2}(s-u) \mathbf{E}_{\Lambda m}^{t}(u) d u=\mathbf{R}(s), \quad s \in \mathbb{R}, \\
& \mathbf{R}(s)=\left\{\begin{array}{cc}
0, & s \in \mathbb{R}^{++}, \\
\int_{-\infty}^{\infty} \mathbf{G}_{2}(s-u) \mathbf{E}_{\Lambda}^{t}(u) d u, & s \in \mathbb{R}^{-},
\end{array}\right.
\end{aligned}
$$

where

$$
\begin{aligned}
& \mathbf{E}_{\Lambda m}^{t}(u)=\left\{\begin{array}{cc}
\mathbf{E}_{m}^{t}(u), & u \in \mathbb{R}^{++}, \\
\Lambda^{t}(u), & u \in \mathbb{R}^{-},
\end{array}\right. \\
& \mathbf{E}_{\Lambda}^{t}(u)=\left\{\begin{array}{cc}
\mathbf{E}^{t}(u), & u \in \mathbb{R}^{++}, \\
\Lambda^{t}(u), & u \in \mathbb{R}^{-},
\end{array}\right.
\end{aligned}
$$

which has a Wiener-Hopf form bearing similarity to (7.12) and can be solved using the same kind of techniques. The solution of (8.14) will be discussed for the scalar case in Sec. 9.

We identify this case as determining the history that yields the maximum free energy associated with the set of equivalent states $\sigma_{R} \in \Sigma_{R}$. This follows (putting $\sigma^{i}$ equal to the zero state) by Theorem 4.4 (see also Remark 4.5). Note that (8.9) in this case ensures that we are optimizing in $\sigma_{R}$. An explicit form of the maximum free energy will be given in later sections for a particular class of materials.

In the remainder of this section, we will show how free energies emerge from the above considerations. The discussion will in fact be generalized in that the specific optimizations outlined above will not be used explicitly, but only the orthogonality condition

$$
\left(\mathbf{E}_{d}^{t}, \mathbf{E}_{1}^{t}\right)=0, \quad \mathbf{E}_{d}^{t}=\mathbf{E}_{1}^{t}-\mathbf{E}^{t}
$$

from which it follows that

$$
\left\|\mathbf{E}^{t}\right\|^{2}=\left\|\mathbf{E}_{1}^{t}\right\|^{2}+\left\|\mathbf{E}_{d}^{t}\right\|^{2} .
$$

Using (8.10), this orthogonality can be shown to hold for the two sets of equations (8.12) and (8.13) (after differentiation with respect to $s$ ), where $\mathbf{E}_{1}^{t}$ is replaced by $\mathbf{E}_{m}^{t}$. It also applies to the solutions that emerge from the more general variational scheme introduced in Sec. 11.

Let us first state the characteristic properties of an isothermal free energy, provable within a general framework $[16,17,7]$ :

P1:

$$
\frac{\partial \psi(t)}{\partial \mathbf{E}(t)}=\mathbf{T}(t) .
$$

P2: Let $\mathbf{E}^{\dagger}$ be a static history equal to $\mathbf{E}_{0}$ at all past times. Then

$$
\psi\left(\mathbf{E}^{\dagger}\right)=\phi\left(\mathbf{E}_{0}\right)
$$

where $\phi\left(\mathbf{E}_{0}\right)$ is the elastic free energy defined by (6.10).

P3: For any history $\mathbf{E}_{a}^{t}$,

$$
\psi\left(\mathbf{E}_{a}^{t}\right) \geq \phi\left(\mathbf{E}_{a}(t)\right)
$$


P4: Condition (3.4) holds. For $\psi$ differentiable, this will be true if

$$
\dot{\psi}(t)=\mathbf{T}(t) \cdot \dot{\mathbf{E}}(t)-D(t),
$$

where $D(t) \geq 0$.

The discussion is restricted to differentiable histories and continuations, except at the current time $t$ where finite discontinuities in $\mathbf{E}_{1}^{t}$ and $\mathbf{E}_{m}^{t}$ must be allowed.

Note that P2 eliminates the freedom of an additive constant in $\psi$, provided $\phi$ is taken to be uniquely defined.

Proposition 8.1. The quantity

$$
\psi(t)=S(t)+\left\|\mathbf{E}_{1}^{t}\right\|^{2},
$$

where $\mathbf{E}_{1}^{t}$ is defined by (8.8) and constrained by (8.9), (8.15), obeys properties P1-P4 of a free energy if $\mathbf{E}_{d}^{t}$ has a finite discontinuity at the origin.

Proof. Property P1 follows from (8.18) on noting that

$$
\frac{\partial S(t)}{\partial \mathbf{E}(t)}=\mathbf{T}(t)
$$

We can write (8.18) in the form

$$
\psi(t)=\phi(t)+\left\|\mathbf{E}_{1}^{t}-\mathbf{E}_{1}^{\dagger}\right\|^{2}
$$

where $\mathbf{E}_{1}^{\dagger}$ is the static history/continuation that $\mathbf{E}_{1}^{t}$ becomes if $\mathbf{E}^{t}$ is the static history equal to $\mathbf{E}(t)$ at each time. It is the static continuation (with a minus sign) in the first case considered above and the static history in the second case. In intermediate cases, it can always be chosen to be the static continuation, using the freedom exposed at the end of Sec. 11. The quantity $\phi(t)$ is the elastic free energy for strain $\mathbf{E}(t)$. Relation (8.19) follows with the aid of (8.7) and (8.10). Note that (8.19) is an explicitly positive form of the free energy. Properties P2, P3 follow immediately.

We may replace $\left\|\mathbf{E}_{1}^{t}\right\|^{2}$ by $\left\|\mathbf{E}^{t}\right\|^{2}-\left\|\mathbf{E}_{d}^{t}\right\|^{2}$ in (8.18) by virtue of (8.16). Differentiating and using the fact that $S(t)+\left\|\mathbf{E}^{t}\right\|^{2}=W(t)$ as given by $(8.3)_{1}$ is also given by $(8.1)_{1}$, we obtain

$$
\begin{aligned}
\dot{\psi}(t) & =\mathbf{T}\left(\mathbf{E}^{t}\right) \cdot \dot{\mathbf{E}}(t)-D(t) \\
D(t) & =\frac{d}{d t}\left\|\mathbf{E}_{d}^{t}\right\|^{2},
\end{aligned}
$$

so that if $\mathrm{P} 4$ is to hold, $D(t)$ must be nonnegative. ${ }^{5}$

We shall treat the general case where $\mathbf{E}_{d}^{t}$ is nonzero on $\mathbb{R}$. The important special case where it is zero on $\mathbb{R}^{--}$is covered by the general demonstration.

It is convenient to use $\mathbf{E}_{d}$ rather than $\mathbf{E}_{d}^{t}$ at this point, the former quantity having a discontinuity at time $t$. Thus

$$
\left\|\mathbf{E}_{d}\right\|^{2}=\frac{1}{2} \int_{-\infty}^{\infty} \int_{-\infty}^{\infty} \mathbf{G}_{12}(s-u) \mathbf{E}_{d}(u) \cdot \mathbf{E}_{d}(s) d u d s
$$

\footnotetext{
${ }^{5}$ We note here that, in a related context [5], the assumption that $\psi$ is differentiable is avoided by using the integrated dissipation inequality, although the argument is less straightforward.
} 
and

$$
\frac{d}{d t}\left\|\mathbf{E}_{d}\right\|^{2}=\int_{-\infty}^{\infty} \mathbf{G}_{12}(t-u) \mathbf{E}_{d}(u) d u \cdot\left(\mathbf{E}_{d}\left(t^{-}\right)-\mathbf{E}_{d}\left(t^{+}\right)\right)
$$

where the symmetry of $\mathbf{G}$ has been used. At $s=0$, differentiating (8.9) with respect to $t$ gives

$$
\int_{-\infty}^{\infty} \mathbf{G}_{12}(t-u) \mathbf{E}_{d}(u) d u=\mathbf{G}^{\prime}(0)\left(\mathbf{E}_{d}\left(t^{+}\right)-\mathbf{E}_{d}\left(t^{-}\right)\right)
$$

so that

$$
\frac{d}{d t}\left\|\mathbf{E}_{d}^{t}\right\|^{2}=-\mathbf{G}^{\prime}(0)\left|\mathbf{E}_{d}\left(t^{-}\right)-\mathbf{E}_{d}\left(t^{+}\right)\right|^{2} \geq 0
$$

In particular, if $\mathbf{E}_{d}^{t}$ vanishes on $\mathbb{R}^{--}$, then $\mathbf{E}_{d}\left(t^{+}\right)=\mathbf{E}_{d}^{t}\left(0^{-}\right)=0$.

The connection between the discontinuity in the history at the current time and the rate of dissipation is interesting from a physical point of view. The minimum free energy given by (6.17) is of course a special case of (8.18) with the appropriate discontinuity at the current time [5].

9. The discrete spectrum model. In the remaining sections, we derive explicit forms of various free energies associated with a discrete spectrum material. Only the scalar case will be considered. There is in fact no difficulty about generalizing much (although not all) of the discussion to include materials for which a number of factorizations of $H(\omega)$ (the scalar version of (7.2)) preserving the singularity structure are possible; in particular, where $H$ as a function of $\omega^{2}$ (see (9.3) below) has only discrete zeros. One result, namely Proposition 9.1, proved later, is in fact generalized in this way. However, in the first presentation of these results, it was judged better, for the sake of clarity, to confine the discussion to discrete spectrum materials.

The stress function $T(t)$ and the current value and strain history $\left(E(t), E^{t}\right)$ are scalars related by

$$
T(t)=G(0) E(t)+\int_{0}^{\infty} G^{\prime}(s) E^{t}(s) d s,
$$

where $G_{0}$ and $G^{\prime}$ are also scalar quantities. The relaxation function

$$
G(t)=G_{0}+\int_{0}^{t} G^{\prime}(u) d u, \quad G_{0}=G(0),
$$

is given by the explicit form

$$
\begin{aligned}
G(t) & =G_{\infty}+\sum_{i=1}^{n} G_{i} e^{-\alpha_{i} t} \\
G_{\infty} & =G_{0}-\sum_{i=1}^{n} G_{i}
\end{aligned}
$$


where $n$ is a positive integer, the inverse decay times $\alpha_{i} \in \mathbb{R}^{+}, i=1,2, \ldots, n$, and the coefficients $G_{i}$ are also generally assumed to be positive. Let $\alpha_{1}<\alpha_{2}<\alpha_{3} \ldots$ We have

$$
G^{\prime}(t)=\sum_{i=1}^{n} g_{i} e^{-\alpha_{i} t}, \quad g_{i}=-\alpha_{i} G_{i}<0 .
$$

Also,

$$
\begin{aligned}
G_{F}^{\prime}(\omega) & =\int_{0}^{\infty} G^{\prime}(s) e^{-i \omega s} d s \\
& =\sum_{i=1}^{n} \frac{g_{i}}{\alpha_{i}+i \omega} \\
& =G_{c}^{\prime}(\omega)-i G_{s}^{\prime}(\omega),
\end{aligned}
$$

where

$$
\begin{aligned}
G_{c}^{\prime}(\omega) & =\sum_{i=1}^{n} \frac{\alpha_{i} g_{i}}{\alpha_{i}^{2}+\omega^{2}}=G_{c}^{\prime}(-\omega) \\
G_{s}^{\prime}(\omega) & =\omega \sum_{i=1}^{n} \frac{g_{i}}{\alpha_{i}^{2}+\omega^{2}}=-G_{s}^{\prime}(-\omega), \\
G_{s}^{\prime}(\omega) & <0 \forall \omega \in \mathbb{R}^{++} .
\end{aligned}
$$

The scalar quantity $H(\omega)$ corresponding to (7.2) has the form

$$
H(\omega)=-\omega^{2} \sum_{i=1}^{n} \frac{g_{i}}{\alpha_{i}^{2}+\omega^{2}} \geq 0, \omega \in \mathbb{R} .
$$

It is shown in [4] that

$$
H(\omega)=H_{\infty} \prod_{i=1}^{n}\left\{\frac{\gamma_{i}^{2}+\omega^{2}}{\alpha_{i}^{2}+\omega^{2}}\right\},
$$

where $H_{\infty}=H(\infty)$, defined by the scalar form of (7.4) to be

$$
H_{\infty}=-G^{\prime}(0)=-\sum_{i=1}^{n} g_{i}>0 .
$$

In (9.4), $\gamma_{1}=0$ and $\left(-\gamma_{i}^{2}\right), i=2,3, \ldots, n$, are the zeros of $H_{1}\left(\omega^{2}\right)=H(\omega)$ that occur such that $\alpha_{1}^{2}<\gamma_{2}^{2}<\alpha_{2}^{2}<\gamma_{3}^{2} \ldots$

The factorization of $H$ as specified by (9.4) may be established by inspection to be

$$
\begin{aligned}
H_{+}(\omega) & =h_{\infty} \prod_{i=1}^{n}\left\{\frac{\omega-i \gamma_{i}}{\omega-i \alpha_{i}}\right\}, \\
H_{-}(\omega) & =h_{\infty} \prod_{i=1}^{n}\left\{\frac{\omega+i \gamma_{i}}{\omega+i \alpha_{i}}\right\} \\
& =\bar{H}_{+}(\omega)=H_{+}(-\omega) ; h_{\infty}=H_{\infty}^{1 / 2} .
\end{aligned}
$$

In this paper, we consider a much larger class of factorizations of $H$ obtained by interchanging the zeros of $H_{+}$and $H_{-}$, but leaving the singularity structure unchanged. There are $N=2^{n-1}$ distinct factorizations of this kind, which we distinguish by the label 
$f=0,1,2, \ldots, N$. The case $f=0$ is where no zeros are interchanged and $f=N$ where all zeros are interchanged. These can be written as

$$
\begin{aligned}
H(\omega) & =H_{+}^{f}(\omega) H_{-}^{f}(\omega) \\
H_{+}^{f}(\omega) & =h_{\infty} \prod_{i=1}^{n}\left\{\frac{\omega-i \rho_{i}}{\omega-i \alpha_{i}}\right\} \\
H_{-}^{f}(\omega) & =h_{\infty} \prod_{i=1}^{n}\left\{\frac{\omega+i \rho_{i}}{\omega+i \alpha_{i}}\right\} \\
& =\bar{H}_{+}^{f}(\omega)=H_{+}^{f}(-\omega), \\
\rho_{i} & =\varepsilon_{i} \gamma_{i}, \quad \varepsilon_{1}=1, \quad \varepsilon_{i}= \pm 1, i=2,3, \ldots, n .
\end{aligned}
$$

The superscript $f$ is omitted on $\rho_{i}$ and is to be understood from the context. Most of the relations derived in [4] do not depend on the location of the zeros of $H_{+}$and $H_{-}$, but do depend crucially on the location of the singularities. We note here certain results to be used later, which can be proved in a manner identical to that given in [4].

The quantity

$$
\begin{aligned}
Q^{(f t)}(\omega) & =H_{-}^{f}(\omega) E_{+}^{t}(\omega) \\
& =q_{-}^{(f t)}(\omega)-q_{+}^{(f t)}(\omega)
\end{aligned}
$$

where $q_{-}^{(f t)}(\omega)$ is analytic in $\Omega^{+}$, goes to zero at large $\omega$ as $\omega^{-1}$, while $q_{+}^{(f t)}(\omega)$ is analytic in $\Omega^{-}$with similar large $\omega$ behaviour. They are given by the limit $z \rightarrow \omega \in \mathbb{R}$ of

$$
q^{(f t)}(z)=\frac{1}{2 \pi i} \int_{-\infty}^{\infty} \frac{Q^{(f t)}(\omega)}{\omega-z} d \omega
$$

from above and below the real axis respectively. The analyticity of these quantities on $\mathbb{R}$ follows from the assumed analyticity of $G_{F}^{\prime}$ and $E_{+}^{t}$ on $\mathbb{R}[4]$. They are defined over the entire complex plane by analytic continuation.

It can be shown that [4]

$$
\begin{aligned}
\frac{d}{d t} q_{+}^{(f t)}(\omega) & =-i \omega q_{+}^{(f t)}(\omega)-K_{f}(t) \\
\frac{d}{d t} q_{-}^{(f t)}(\omega) & =-i \omega q_{-}^{(f t)}(\omega)-K_{f}(t)+H_{-}^{f}(\omega) E(t) \\
K_{f}(t) & =\frac{1}{2 \pi} \int_{-\infty}^{\infty} H_{-}^{f}(\omega) E_{+}^{t}(\omega) d \omega+\frac{1}{2} h_{\infty} E(t) \\
& =\frac{1}{2 \pi} \int_{-\infty}^{\infty} H_{-}^{f}(\omega)\left[E_{+}^{t}(\omega)-\frac{E(t)}{i \omega^{-}}\right] d \omega
\end{aligned}
$$

using the notation of (7.6). Also,

$$
\begin{aligned}
& \lim _{|\omega| \rightarrow \infty} \omega q_{+}^{(f t)}(\omega)=i K_{f}(t), \\
& \lim _{|\omega| \rightarrow \infty} \omega q_{-}^{(f t)}(\omega)=i\left(K_{f}(t)-h_{\infty} E(t)\right)
\end{aligned}
$$


and

$$
\begin{aligned}
& \frac{1}{2 \pi} \int_{-\infty}^{\infty} q_{+}^{(f t)}(-\omega) d \omega=-\frac{1}{2} K_{f}(t)=\frac{1}{2 \pi} \int_{-\infty}^{\infty} q_{+}^{(f t)}(\omega) d \omega \\
& \frac{1}{2 \pi} \int_{-\infty}^{\infty} q_{-}^{(f t)}(-\omega) d \omega=\frac{1}{2}\left(K_{f}(t)-h_{\infty} E(t)\right)=\frac{1}{2 \pi} \int_{-\infty}^{\infty} q_{-}^{(f t)}(\omega) d \omega
\end{aligned}
$$

We also consider a generalization of (9.6) where the strain is defined over $\mathbb{R}$, with Fourier transforms given by (8.6). With a view to future applications, the relevant relations will be written for $E_{d}^{t}$, the scalar version of the quantity introduced in (8.8):

$$
\begin{aligned}
U^{(f t)}(\omega) & =H_{-}^{f}(\omega) E_{d F}^{t}(\omega)=H_{-}^{f}(\omega)\left(E_{d+}^{t}(\omega)+E_{d-}^{t}(\omega)\right) \\
& =u_{-}^{(f t)}(\omega)-u_{+}^{(f t)}(\omega),
\end{aligned}
$$

where

$$
\begin{aligned}
& u_{ \pm}^{(f t)}(\omega)=\lim _{z \rightarrow \omega \mp} u^{(f t)}(z), \\
& u^{(f t)}(z)=\frac{1}{2 \pi i} \int_{-\infty}^{\infty} \frac{U^{(f t)}(\omega)}{\omega-z} d \omega .
\end{aligned}
$$

The quantities $u_{ \pm}^{(f t)}$ have the same analyticity properties as $q_{ \pm}^{(f t)}$. It is clear that

$$
\begin{aligned}
& u_{-}^{(f t)}(\omega)=q_{d-}^{(f t)}+H_{-}^{f}(\omega) E_{d-}^{t}(\omega)=q_{d+}^{(f t)}+H_{-}^{f}(\omega) E_{d F}^{t}(\omega) \\
& u_{+}^{(f t)}(\omega)=q_{d+}^{(f t)}
\end{aligned}
$$

where $q_{d \pm}^{(f t)}$ are defined by $(9.6)$ and (9.7) with $E_{d+}^{t}$ replacing $E_{+}^{t}$.

We will now prove a result that is not confined to a discrete spectrum material. It relies upon the fact that $H(\omega)$ is factorizable into quantities analytic on $\Omega^{+}$and $\Omega^{-}$, with zeros that may be interchanged. Analyticity excludes non-isolated as well as isolated singularities, of course. It relies also on the analyticity of the various quantities on the real axis

Proposition 9.1. The strain history and continuation $E_{d}^{t}$ obeys (8.9) if $u_{-}^{(f t)}$ vanishes. Relation (8.9) implies that $u_{-}^{(f t)}$ vanishes in two cases: (i) where no exchange of zeros in $H_{ \pm}$takes place $(f=0)$; and (ii) where $E_{d-}^{t}$ vanishes.

Proof. Applying Plancherel's theorem to (8.9) gives in the scalar case

$$
\int_{-\infty}^{\infty} \frac{H(\omega)}{\omega}\left(E_{d+}^{t}(\omega)+E_{d-}^{t}(\omega)\right) e^{i \omega s} d \omega=0, \quad s \in \mathbb{R}^{-},
$$

by virtue of the scalar form of (7.5). With the aid of (9.11), we can write (9.14) in the form

$$
\int_{-\infty}^{\infty} \frac{H_{+}^{f}(\omega)}{\omega}\left(u_{-}^{(f t)}(\omega)-u_{+}^{(f t)}(\omega)\right) e^{i \omega s} d \omega=0, \quad s \in \mathbb{R}^{-}
$$


where $H_{+}^{f}(\omega)$ vanishes linearly at $\omega=0$. Closing the integral involving $u_{+}^{(f t)}$ on $\Omega^{-}$, we see that it vanishes by Cauchy's theorem, so that the condition becomes

$$
F(s)=\int_{-\infty}^{\infty} \frac{H_{+}^{f}(\omega)}{\omega} u_{-}^{(f t)}(\omega) e^{i \omega s} d \omega=0, \quad s \in \mathbb{R}^{-}
$$

It is trivially satisfied if $u_{-}^{(f t)}$ vanishes, which is the first assertion. We now prove that, in the two specified cases, if $(9.15)$ is satisfied, then $u_{-}^{(f t)}$ is zero. The function $F(s)$ vanishes on $\mathbb{R}^{-}$, so that its Fourier transform $2 \pi\left(H_{+}^{f}(\omega) / \omega\right) u_{-}^{(f t)}(\omega)$ must be analytic in $\Omega^{(-)}$. This can only be true if $H_{+}^{f}(\omega) / \omega$ vanishes at each of the isolated singularities of $u_{-}^{(f t)}$ in $\Omega^{(-)}$; and for each cut type singularity in $u_{-}^{(f t)}$, we must have a compensating singularity of the same kind in $H_{+}^{f}(\omega) / \omega$. Since $H_{+}^{f}(\omega) / \omega$ is analytic in $\Omega^{(-)}, u_{-}^{(f t)}$ cannot have cuts in this half-plane. As for isolated singularities, we distinguish the case where no permutation of zeros has taken place $(f=0)$ from all others.

For the case $f=0, H_{+}$has no zeros in $\Omega^{(-)}$. The only remaining option is that $u_{-}^{(f t)}$ has no singularities in $\Omega^{(-)}$and therefore in $\Omega$. It vanishes at infinity as $\omega^{-1}$, and therefore, by Liouville's theorem, vanishes everywhere.

If permutations of zeros are allowed, then $H_{+}^{f}$ has zeros in $\Omega^{(-)}$. Consider the definition of $u_{-}^{(f t)}$ given by (9.11) and (9.12). Evaluating the integral by closing the contour on $\Omega^{(-)}$shows that $u_{-}^{(f t)}$ has singularities in $\Omega^{(-)}$where $H_{-}^{f}$ and $E_{d-}^{t}$ have singularities, and these must be isolated, as concluded above. If a zero in $H_{+}^{f}$ cancels a singularity in $H_{-}^{f}$ then, without permutation, the zero and the singularity would cancel in $H$. In other words, there would by no singularity or zero to begin with. Therefore, the only option is that $u_{-}^{(f t)}$ is free of singularities due to $H_{-}^{f}$. It could still have singularities due to $E_{d-}^{t}(\omega)$ cancelled by zeros in $H_{+}^{f}$. However, if $E_{d-}^{t}$ is zero, this cannot be su. Thus, by Liouville's theorem, $u_{-}^{(f t)}$ must be zero.

It will become clear in Sec. 11 that the first case $(f=0)$ corresponds to the minimum free energy, while, from an argument after (9.23) below, case (ii) corresponds to the maximum free energy $(f=N)$. In fact, we see from (9.13) that $E_{d-}^{t}$ vanishing yields $q_{d-}^{(N t)}=0$ so that Proposition 9.1 amounts to stating that condition (8.9) implies that $q_{d-}^{(N t)}$ vanishes. Recalling that (8.9) in this case is the condition for equivalence of states $E_{1}^{t}$ and $E^{t}$, we conclude that $q_{1-}^{(N t)}$ defined by (9.6), for a given history $E_{1}^{t}$, is a state variable on the space of minimal states $\Sigma_{R}$.

In Sec. 11, we shall adopt the condition $u_{-}^{(f t)}=0$ as the constraint on our variational scheme in all cases, not just in the two described in Proposition 9.1. This condition implies (8.9) but is a stronger assumption in general (except in the two cases), which is in effect the content of Proposition 9.1.

Observe that by applying the argument leading to (9.15) to the scalar form of (7.11), using (9.6), the stress function at time $t$ can be written as

$$
T(t)=G_{0} E(t)+\frac{1}{\pi i} \int_{-\infty}^{\infty} \frac{H_{+}^{f}(\omega)}{\omega} q_{-}^{(f t)}(\omega) d \omega
$$


for each permutation $f$. All of these are of course equivalent. It is proved in [5], for general materials and in the full tensorial case, that (stated for scalars) $q_{-}^{(f t)}$ is a state variable for $f=0$. The argument used in that reference can easily be extended to all $f$. Briefly, it amounts to showing that if $F(s)$, given by $(9.15)$, but with $u_{-}^{(f t)}$ replaced by $q_{d-}^{(f t)}\left(E_{d}\right.$ in the scalar version of $(8.9)$ zero on $\mathbb{R}^{-}$), vanishes on $\mathbb{R}^{-}$, then $q_{d-}^{(f t)}$ is zero. This follows by the argument used in case (ii) of Proposition 9.1. Thus, we state

Corollary 9.2. The quantity $q_{-}^{(f t)}$ is a function of state for all permutations $f$.

If $q_{-}^{(f t)}$ is a function of state, then in general $q_{+}^{(f t)}=q_{-}^{(f t)}-H_{-}^{f} E_{+}^{t}$ will not be, because of the occurrence of the transformed history.

It is convenient to introduce a conventional scalar product

$$
\langle f, g\rangle=\frac{1}{2 \pi} \int_{-\infty}^{\infty} \bar{f}(\omega) g(\omega) d \omega
$$

as well as (8.5). Observe that $f$ and $g$ are orthogonal in this scalar product if $f$ is analytic in $\Omega^{+}$and $g$ in $\Omega^{-}$or vica versa and both vanish at infinity at least as strongly as $\omega^{-1}$. In this notation, (9.16) becomes

$$
T(t)=G_{0} E(t)+\left\langle h, q_{-}^{(f t)}\right\rangle, \quad h(\omega)=2 i \frac{H_{-}^{f}(\omega)}{\omega} .
$$

We write

$$
\langle f, f\rangle=\|f\|_{L}
$$

indicating the Lesbeque $L^{2}$ norm.

Let us consider further the solution of (8.14) in the scalar case. The argument is confined to discrete spectrum models. We choose the factorization $f=N$ corresponding to all zeros interchanged, which will be justified later. First note that $E_{m}^{t}$ and $E^{t}$ are equivalent states so that, by Corollary 9.2 ,

$$
q_{d-}^{(N t)}(\omega)=q_{m-}^{(N t)}(\omega)-q_{-}^{(N t)}(\omega)=0
$$

where $q_{m-}^{(N t)}(\omega)$ is defined by (9.6) and (9.7) but with $E_{m+}^{t}$ replacing $E_{+}^{t}$. It follows (see (9.6)) that

$$
E_{d+}^{t}(\omega)=-\frac{q_{d+}^{(N t)}(\omega)}{H_{-}^{N}(\omega)} .
$$

Now, taking the Fourier transform of (8.14) and using (9.6), we obtain

$$
2 i H_{+}^{N}(\omega)\left(q_{m-}^{(N t)}(\omega)-q_{m+}^{(N t)}(\omega)+H_{-}^{N}(\omega) \Lambda_{-}^{t}(\omega)\right)=-\omega R_{-}(\omega),
$$

where the subscripts on $\Lambda_{-}^{t}$ and $R_{-}$indicate that these are Fourier transforms of quantities that are zero on $\mathbb{R}^{++}$and therefore analytic in $\Omega^{+}$. The explicit form of $R_{-}(\omega)$ is

$$
\begin{aligned}
R_{-}(\omega) & =-\frac{2 i}{\omega}\left[H_{+}^{N}(\omega)\left(q_{-}^{(N t)}(\omega)-q_{+}^{(N t)}(\omega)+H_{-}^{N}(\omega) \Lambda_{-}^{t}(\omega)\right)\right]_{-} \\
& =-\frac{2 i}{\omega}\left[H_{+}^{N}(\omega)\left(q_{-}^{(N t)}(\omega)+H_{-}^{N}(\omega) \Lambda_{-}^{t}(\omega)\right)\right]_{-},
\end{aligned}
$$


where the notation $[\cdot]_{-}$indicates that the corresponding time domain function was put equal to zero on $\mathbb{R}^{++}$. The bracketed factor in (9.21) goes to zero as $\omega^{-1}$ at large $\omega$.

We now divide $(9.20)$ by $2 i H_{+}^{N}$, remembering that the zeros in this quantity are in $\Omega^{(-)}$so that the ratio

$$
V_{-}(\omega)=-\frac{\omega}{2 i} \frac{R_{-}(\omega)}{H_{+}^{N}(\omega)}
$$

is analytic in $\Omega^{+}$. We conclude from (9.20) and Liouville's theorem that

$$
\begin{aligned}
q_{m+}^{(N t)}(\omega) & =0 \\
q_{-}^{(N t)}(\omega)+H_{-}^{N}(\omega) \Lambda_{-}^{t}(\omega) & =V_{-}(\omega)
\end{aligned}
$$

since all quantities go to zero at infinity. The first relation of (9.22) is the important one. The second becomes, with the aid of (9.21), using a similar notational convention,

$$
\left[H_{+}^{N}(\omega)\left(q_{-}^{(N t)}(\omega)+H_{-}^{N}(\omega) \Lambda_{-}^{t}(\omega)\right)\right]_{+}=0
$$

which is the equation for $\Lambda_{-}^{t}$. It will not be explored further.

Equation $(9.22)_{1}$ gives that

$$
q_{d+}^{(N t)}(\omega)=-q_{+}^{(N t)}(\omega)
$$

and (9.19) becomes

$$
E_{d+}^{t}(\omega)=E_{m+}^{t}(\omega)-E_{+}^{t}(\omega)=\frac{q_{+}^{(N t)}(\omega)}{H_{-}^{N}(\omega)}
$$

so that

$$
E_{m+}^{t}(\omega)=\frac{q_{-}^{(N t)}(\omega)}{H_{-}^{N}(\omega)},
$$

which is the desired solution. For discrete sprectrum materials, the singularities in $H_{-}^{N}$ cancel those in $q_{-}^{(N t)}$, as may be seen by closing the contour in the definition of $q_{-}^{(f t)}$ on $\Omega^{-}$. Thus, because the zeros of $H_{-}^{N}$ are all in $\Omega^{(+)}$, we see that $E_{m+}^{t}$ has the correct singularity structure and that our initial adoption of the factorization $f=N$ was justified.

The form of the free energy associated with $E_{m-}^{t}$ is given by the scalar version of (8.18) with $E_{m-}^{t}$ replacing $E_{1}^{t}$. Using $(9.23)$, this can be written as

$$
\psi^{(M)}(t)=S(t)+\left\|q_{-}^{(N t)}\right\|_{L}^{2},
$$

which is the maximum free energy associated with the minimal state $\sigma_{R}$.

10. Free energies as discrete quadratic forms. Substituting $G^{\prime}(s)$ given by $(9.2)$ into (9.1), evaluated at $t+u, u \leq 0$, we see that two states are equivalent in the sense of (6.8) if and only if the difference between the states, denoted by $E_{d}^{t}$, has the property that

$$
\begin{aligned}
E_{d}^{(i)}(t) & =\int_{0}^{\infty} e^{-\alpha_{i} s} E_{d}^{t}(s) d s=E_{d+}^{t}\left(-i \alpha_{i}\right)=0 \\
E_{d}(t) & =0
\end{aligned}
$$


It follows that free energies formed by quadratic forms of these quantities, for a given history, are functions of state in the sense of $[1,8,9]$. Such forms of the free energy have been studied by Graffi and Fabrizio [18, 19] and Fabrizio et al [20]. We summarize some results here for later use. Define a vector $\mathbf{e}$ in $\mathbb{R}^{n}$ with components

$$
\begin{aligned}
e_{i}(t) & =E(t)-\alpha_{i} E_{i}(t)=\dot{E}_{i}(t), \\
E_{i}(t) & =E_{+}^{t}\left(-i \alpha_{i}\right),
\end{aligned}
$$

where (7.8) has been used. Consider the quantity

$$
\psi(t)=\phi(t)+\frac{1}{2} \mathbf{e}^{\top} \mathbf{C e},
$$

where $\phi(t)$ is the elastic free energy, defined by the scalar version of $(6.10)$, and $\mathbf{C}$ is a symmetric, positive definite matrix with components $C_{i j}, i, j=1,2, \ldots, n$. It is clear that $\psi(t)$ has property P3 (Sec. 8) of a free energy.

For a stationary history $E^{t}(s)=E(t), s \in \mathbb{R}^{+}$, we have $E_{i}(t)=E(t) / \alpha_{i}$ (see (7.6) for a constant history) so that $\mathbf{e}_{i}(t)=0, i=1,2, \ldots, n$. Therefore, $\psi(t)$ has property P2 of a free energy. Condition (8.17) requires that

$$
\sum_{j=1}^{n} C_{i j}=G_{i}, \quad i=1,2, \ldots, n .
$$

It is easy to show that

$$
\begin{aligned}
\dot{\psi}(t)+D(t) & =T(t) \dot{E}(t), \\
D(t) & =\frac{1}{2} \mathbf{e}^{\top} \Gamma \mathbf{e}, \\
\Gamma_{i j} & =\left(\alpha_{i}+\alpha_{j}\right) C_{i j},
\end{aligned}
$$

where $\Gamma_{i j}$ are the elements of the matrix $\Gamma$. The final requirement $\mathrm{P} 4$ is that $D(t) \geq 0$ so that $\Gamma$ must be positive definite.

The forms of $\mathbf{C}$ and $\Gamma$ corresponding to the minimum free energy can be deduced without difficulty from formulae given in [4]. Other forms are derived in Sec. 11.

The set of free energies expressible in the form (10.2), where $\mathbf{C}$ has the required positivity properties, is identical to the set $\mathcal{S}_{\sigma}$ of free energies associated with the equivalence class $\sigma_{R}$, which are functions of state. This is a convex set, by virtue of a result [7] that any set of free energies, associated with a given history and current value, has this property. The results of the next sections will provide a family of members of this set.

11. A family of free energies in $\Sigma_{R}$. We will now derive an expression for free energies corresponding to each permutation $f$ and a given state $\sigma=\left(E(t), E^{t}\right)$, which are functionals only of the equivalence class $\sigma_{R}$ rather than $\sigma$. This family includes the minimum free energy derived in [4], a maximum free energy defined on $\Sigma_{R}$ (not the work function given by (8.1) which was referred to as the maximum free energy in [4] but rather (9.24)) and intermediate functionals. No clear ordering emerges from the general treatment. However, in Sec. 12, we present explicit forms for these free energies and establish a partial ordering within the family. 
These developments are the frequency domain generalization of the theory presented in Sec. 8. In frequency space, we shall see that it is possible to obtain explicit results with relative ease.

The following constrained optimization problem is considered. For a given state $\left(E(t), E^{t}\right)$, we seek $E_{d}^{t}$ that minimizes $\left\|E_{1}^{t}\right\|^{2}$, where $E_{1}^{t}=E^{t}+E_{d}^{t}$, subject not to (8.9) but to the stronger condition (except in two cases; see Proposition 9.1)

$$
u_{-}^{(f t)}(\omega)=\frac{1}{2 \pi i} \int_{-\infty}^{\infty} \frac{H_{-}^{f}\left(\omega^{\prime}\right) E_{d F}^{t}\left(\omega^{\prime}\right)}{\omega^{\prime}-\omega^{+}} d \omega^{\prime}=0 .
$$

Note that, in general, $E_{1}^{t}, E_{d}^{t}$ are nonzero on $\mathbb{R}$. It follows from (9.13) that

$$
E_{d F}^{t}(\omega)=-\frac{q_{d+}^{(f t)}(\omega)}{H_{-}^{f}(\omega)}=-\frac{1}{2 \pi i H_{-}^{f}(\omega)} \int_{-\infty}^{\infty} \frac{H_{-}^{f}\left(\omega^{\prime}\right) E_{d F}^{t}\left(\omega^{\prime}\right)}{\omega^{\prime}-\omega^{-}} d \omega^{\prime}
$$

Using the factorization (9.5), we see that the quantity to be minimized has the form

$$
M(t)=\left\|H_{-}^{f}\left(E_{+}^{t}+E_{d F}^{t}\right)\right\|_{L}^{2} .
$$

Now

$$
U^{(f t)}(\omega)=H_{-}^{f}(\omega) E_{d F}^{t}(\omega)=-u_{+}^{(f t)}(\omega)
$$

by virtue of (11.1). Using (9.6), we see that

$$
M(t)=\left\|q_{-}^{(f t)}-q_{+}^{(f t)}-u_{+}^{(f t)}\right\|_{L}^{2} .
$$

Let us vary $u_{+}^{(f t)}(\omega)$, replacing it by $u_{+}^{(f t)}(\omega)+k(\omega)$ where $k(\omega)$ is arbitrary except for the constraint that it has the same analytic structure as $u_{+}^{(f t)}(\omega)$-namely that it is analytic in $\Omega^{-}$and goes to zero at infinity as $\omega^{-1}$. The minimization condition is

$$
\left\langle k, q_{-}^{(f t)}-q_{+}^{(f t)}-u_{+}^{(f t)}\right\rangle=0
$$

for all allowable $k$. The $q_{-}^{(f t)}$ term drops out by virtue of the comment after (9.17). Application of the corresponding argument in [4] yields that $u_{+}^{(f t)}=-q_{+}^{(f t)}$ or from (9.6), (9.7) and (11.3),

$$
E_{d F}^{(f t)}(\omega)=\frac{q_{+}^{(f t)}(\omega)}{H_{-}^{f}(\omega)}=\frac{1}{2 \pi i H_{-}^{f}(\omega)} \int_{-\infty}^{\infty} \frac{H_{-}^{f}\left(\omega^{\prime}\right) E_{+}^{t}\left(\omega^{\prime}\right)}{\omega^{\prime}-\omega^{-}} d \omega^{\prime}=E_{d m}^{(f t)}(\omega),
$$

which is the optimal choice. From (11.2) it follows that

$$
q_{1+}^{(f t)}(\omega)=\frac{1}{2 \pi i} \int_{-\infty}^{\infty} \frac{H_{-}^{f}\left(\omega^{\prime}\right) E_{1}^{t}\left(\omega^{\prime}\right)}{\omega^{\prime}-\omega^{-}} d \omega^{\prime}=0 .
$$

The resulting free energy follows by inserting (11.5) (in the form $u_{+}^{(f t)}=-q_{+}^{(f t)}$ ) into (11.4) and recalling (8.18):

$$
\begin{aligned}
\psi_{f}(t) & =S(t)+\left\|q_{-}^{(f t)}\right\|_{L}^{2} \\
& =S(t)+\left\|E_{f}^{t}\right\|^{2}
\end{aligned}
$$


where $E_{f}^{t}$ is the optimal choice of $E_{1}^{t}$ (denoted in the time domain by $E_{m}^{t}$ in (8.12), (8.13) and by $E_{f o}^{t}$ in this context), here given by

$$
E_{f}^{t}(\omega)=\frac{q_{-}^{(f t)}(\omega)}{H_{-}^{f}(\omega)}=\frac{1}{2 \pi i H_{-}^{f}(\omega)} \int_{-\infty}^{\infty} \frac{H_{-}^{f}\left(\omega^{\prime}\right) E_{+}^{t}\left(\omega^{\prime}\right)}{\omega^{\prime}-\omega^{+}} d \omega^{\prime} .
$$

We write

$$
E_{f}^{t}(\omega)=E_{f+}^{t}(\omega)+E_{f-}^{t}(\omega),
$$

where $E_{f+}^{t}(\omega)$ is analytic in $\Omega^{-}$and corresponds to a history before time $t$, while $E_{f-}^{t}(\omega)$ is analytic in $\Omega^{+}$and corresponds to a continuation after time $t$. Formal expressions can be given for these two quantities by using the device introduced in (9.6) and (9.7). However, it will emerge that such a general technique is unnecessary.

If the zeros in $H_{-}^{f}$ are not permuted $(f=0)$, then $E_{f+}^{t}$ vanishes. This case is readily identified as the minimum free energy derived in [4] with $E_{f}^{t}$ identified as the negative of the optimal continuation given in that paper. If all the zeros are permuted $(f=N)$, then $E_{f-}^{t}$ vanishes. To see this, we need to recall the argument used after (9.23), giving that the integral in (11.7) picks out the singularities due to $H_{-}^{f}$. These are then cancelled by the singularities of $H_{-}^{f}$ in the denominator so that the final singularities of $E_{f}^{t}$ are at the zeros of $H_{-}^{f}$. The case where $E_{f-}^{t}$ vanishes, corresponding to the maximum free energy, has $E_{f}^{t}$ as an optimal history up to time $t$. This is the solution given by (9.23) with associated free energy given by (9.24). If some zeros are permuted, we have an intermediate situation where neither $E_{f+}^{t}$ nor $E_{f-}^{t}$ vanish.

Consider the stress corresponding to $E_{f}^{t}$ as given by (8.7):

$$
\begin{aligned}
T(t) & =G_{0} E(t)+T_{1}(t), \\
T_{1}(t) & =-2\left(l, E_{f+}^{t}+E_{f-}^{t}\right)=-2\left(l, E_{f+}^{t}-\bar{E}_{f-}^{t}\right), \\
l(\omega) & =\frac{1}{i \omega}, \quad E_{f-}^{t}(\omega)=E_{f-}^{t}(-\omega),
\end{aligned}
$$

where the evenness of $H$ has been used. Now $E_{f-}^{t}(-\omega)$ is analytic in $\Omega^{-}$and corresponds to the time-reversed history of $E_{f-}^{t}(\omega)$. In this manner, we can give $T(t)$ physical meaning, because of course, stress is a functional of a history defined on $\mathbb{R}^{+}$. This suggests that we can turn all $E_{f}^{t}(\omega), \omega \in \mathbb{R}$, into equivalent histories up to time $t$ corresponding to the Fourier transform of $E_{f+}^{t}(\omega)-E_{f-}^{t}(-\omega)$. This will be so provided that

$$
\left\|E_{f+}^{t}+E_{f-}^{t}\right\|^{2}=\left\|E_{f+}^{t}-\bar{E}_{f-}^{t}\right\|^{2}
$$

which is true if

$$
\begin{aligned}
\left(E_{e+}^{t}, E_{e-}^{t}\right) & =0 \\
E_{e \pm}^{t}(\omega) & =\frac{1}{2}\left(E_{f \pm}^{t}(\omega)+E_{f \pm}^{t}(-\omega)\right)
\end{aligned}
$$

or that the even parts of $E_{f \pm}^{t}(\omega)$ are orthogonal. This condition is trivially satisfied if either $E_{f+}^{t}$ or $E_{f-}^{t}$ vanishes.

It is not in general true in other cases; so we cannot avoid using $E_{f}^{t}$ defined on $\mathbb{R}$ rather than on $\mathbb{R}^{+}$. It is easy to express $\psi_{f}$ in terms of the scalar product (8.5) and a 
history up to time $t$. Observe that

$$
\begin{aligned}
\left|q_{-}^{(f t)}(\omega)\right|^{2} & =H(\omega)\left|E_{f g}^{t}(\omega)\right|^{2}, \\
E_{f g}^{t}(\omega) & =\frac{q_{-}^{(f t)}(\omega)}{H_{-}^{g}(\omega)},
\end{aligned}
$$

where $g$ is any permutation of the zeros. In particular, we can choose $g=N$, namely the case where all zeros are interchanged. All zeros of $H_{-}^{N}$ are in $\Omega^{+}$and $E_{f N}^{t}$ corresponds to a history up to time $t$. However, this history is not equal to the history defining the stress, namely $E_{f+}^{t}(\omega)-E_{f-}^{t}(-\omega)$.

For a static history (see $(7.6)), E_{+}^{t}(\omega)=E_{s}^{t}(\omega)=E(t) /(i \omega)$, we have from (11.7), closing on $\Omega^{(+)}$, that [4]

$$
E_{f s}^{t}(\omega)=\frac{E(t)}{i \omega} .
$$

One can write (11.6) in the form (see (8.19))

$$
\psi_{f}(t)=\phi(t)+\left\|E_{f}^{t}-E_{f s}^{t}\right\|^{2}
$$

on using the scalar form of relation (8.7) and

$$
\left(l, E_{+}^{t}-E_{f}^{t}\right)=\frac{1}{2}\left\langle h, q_{+}^{(f t)}\right\rangle=0,
$$

where $h$ is defined in (9.18) and $l$ in (11.9). The orthogonality follows from the remark after (9.17).

Observe that we have omitted moving $\omega$ off the real axis in (11.10). It does not matter because such poles are cancelled by the $\omega^{2}$ dependence of $H(\omega)$ near the origin.

It is clear that properties P1-P3 of a free energy follow from (11.6) and (11.11). All that remains is to show that

$$
T(t) \dot{E}(t)=\dot{W}(t)=\dot{\psi}_{f}(t)+D_{f}(t) ; \quad D_{f}(t) \geq 0 .
$$

Using (9.6) and the scalar form of (8.4), we can write

$$
\begin{aligned}
W(t) & =S(t)+\left\|q_{-}^{(f t)}-q_{+}^{(f t)}\right\|_{L}^{2} \\
& =S(t)+\left\|q_{-}^{(f t)}\right\|_{L}^{2}+\left\|q_{+}^{(f t)}\right\|_{L}^{2},
\end{aligned}
$$

where the orthogonality of $q_{-}^{(f t)}, q_{+}^{(f t)}$ follows from the remark after (9.17). We note in passing here that this orthogonality is that expressed by (8.15) as may be seen from (11.5) and (11.7). Comparing (11.12) and (11.13), we see with the help of (11.6) that

$$
\begin{aligned}
D_{f}(t) & =\frac{d}{d t}\left\|q_{+}^{(f t)}\right\|^{2}=K_{f}^{2}(t), \\
K_{f}(t) & =\left\langle H_{+}^{(f)}, E_{+}^{t}-E_{s}^{t}\right\rangle,
\end{aligned}
$$

where $E_{s}^{t}$ is the static history $E(t) /(i \omega)$. We have used (9.8) and (9.10) in writing (11.14). The quantity $K_{f}$ is real and $D_{f}$ is clearly nonnegative. 
Let $E_{f o}^{t}(s), s \in \mathbb{R}$, be the time-domain history and continuation, corresponding to $E_{f}^{t}(\omega)$, which has minimum norm. We have

$$
\begin{aligned}
E_{f o}^{t}(s) & =E_{f o+}^{t}(s), \quad s>0, \\
& =E_{f o-}^{t}(s), \quad s<0 ; \\
E_{f o+}^{t}(s)=\left\langle X^{s}, E_{f+}^{t}\right\rangle ; & E_{f o-}^{t}(s)=\left\langle X^{s}, E_{f-}^{t}\right\rangle ; \\
X^{s}(\omega)= & e^{-i \omega s},
\end{aligned}
$$

where $E_{f o+}^{t}$ is zero on $\mathbb{R}^{--}$and $E_{f o-}^{t}$ on $\mathbb{R}^{++}$and their inverse Fourier transforms have been expressed as scalar products.

Recalling (7.9) and (7.10) with similar relations for future continuations, we write

$$
\begin{aligned}
E_{f o \pm}^{t}( \pm \infty) & = \pm i \lim _{\omega \rightarrow 0} \omega E_{f \pm}^{t}(\omega), \\
E_{f o \pm}^{t}\left(0^{ \pm}\right) & = \pm i \lim _{\omega \rightarrow \infty} \omega E_{f \pm}^{t}(\omega) .
\end{aligned}
$$

Now the quantities $E_{f o \pm}^{t}( \pm \infty)$ are generally nonzero. In fact, from (11.7) and (11.15) we see that their difference is given by [4]

$$
\begin{aligned}
E_{f o+}^{t}(\infty)-E_{f o-}^{t}(-\infty) & =\frac{1}{\kappa^{(f)}(0)}\left\langle\kappa^{(f)}, E_{+}^{t}\right\rangle, \\
\kappa^{(f)}(\omega) & =\frac{H_{-}^{f}(\omega)}{\omega} .
\end{aligned}
$$

From (9.9) and (11.7), we deduce that

$$
E_{f}^{t}(\omega) \underset{\omega \rightarrow \infty}{\longrightarrow} \frac{E(t)-K_{f}(t) / h_{\infty}}{i \omega}
$$

implying that

$$
E_{f o+}^{t}\left(0^{+}\right)-E_{f o-}^{t}\left(0^{-}\right)=E(t)-\frac{K_{f}(t)}{h_{\infty}} .
$$

There is therefore a discontinuity in the time domain representation of (11.5), which we denote by $E_{d o}^{(f t)}(s)=E_{f o}^{t}(s)-E^{t}(s)$, of magnitude $K_{f}(t) / h_{\infty}$, at the origin. Recalling (11.14), we see that there is agreement with (8.20).

In the case of the minimum free energy, $E_{f o+}^{t}\left(0^{+}\right)$is zero and we obtain the result given in [4], identifying $\left(-E_{f o+}^{t}\right)$ as the optimal continuation. There is a discontinuity of magnitude $K_{f}(t) / h_{\infty}$ at the origin. In the case of the maximum free energy, $E_{f o+}^{t}\left(0^{-}\right)$ is zero and there is a jump of magnitude $K_{f}(t) / h_{\infty}$ in the optimal history at the origin, as required by $(8.20)$.

Equations (11.16) and (11.17) leave $E_{f o \pm}^{t}$ arbitrary to within an additive constant, in the intermediate cases, which we resolve by adding an extra constraint, apparently without physical significance

$$
E_{f o+}^{t}\left(0^{+}\right)+E_{f o-}^{t}\left(0^{-}\right)=b E(t)
$$

where $b$ is chosen to be some convenient value. This freedom allows us to make a particular choice of $E_{f s}^{t}$ in $(11.11)_{1}$, as pointed out after (8.19). There is no need for such a condition in the two extreme cases. 
Finally, observe that (11.11) can be written in the form

$$
\begin{aligned}
\psi_{f}(t) & =\phi(t)+\left\|p_{f}\right\|_{L}^{2} \\
p_{f}(\omega) & =q_{-}^{(f t)}(\omega)-\frac{H_{-}^{f}(\omega) E(t)}{i \omega} \\
& =\frac{1}{2 \pi i} \int_{-\infty}^{\infty} \frac{H_{-}^{(f)}\left(\omega^{\prime}\right)\left(E_{+}^{t}\left(\omega^{\prime}\right)-\frac{E(t)}{i \omega^{\prime}}\right)}{\omega^{\prime}-\omega^{+}} d \omega^{\prime}
\end{aligned}
$$

It now manifestly depends only on the couple $\left(E(t), q_{-}^{(f t)}\right)$ and is, therefore, a state variable.

12. Explicit forms. Detailed expressions for these free energies are now derived in terms of the viscoelastic parameters of the discrete spectrum model. Also, a ranking will be demonstrated among subsequences of these forms. It is furthermore pointed out that linear combinations of the free energy, with positivity restrictions on the coefficients, are also free energies.

The developments corresponding to those described in [4] go through with little change in the present case; so detailed proofs are omitted.

The quantities $q_{ \pm}^{(f t)}(\omega)$ defined by (9.6) and (9.7) have the form

$$
\begin{aligned}
q_{-}^{(f t)}(\omega) & =i h_{\infty} \sum_{i=1}^{n} \frac{R_{i}^{f} E_{+}^{t}\left(-i \alpha_{i}\right)}{\omega+i \alpha_{i}} \\
q_{+}^{(f t)}(\omega) & =q_{-}^{(f t)}(\omega)-H_{-}^{f}(\omega) E_{+}^{t}(\omega) \\
& =i h_{\infty} \sum_{i=1}^{n} \frac{R_{i}^{f}\left[E_{+}^{t}\left(-i \alpha_{i}\right)-E_{+}^{t}(\omega)\right]}{\omega+i \alpha_{i}}-h_{\infty} E_{+}^{t}(\omega), \\
R_{i}^{f} & =\left(\rho_{i}-\alpha_{i}\right) \prod_{\substack{j=1 \\
j \neq i}}^{n}\left\{\frac{\rho_{j}-\alpha_{i}}{\alpha_{j}-\alpha_{i}}\right\},
\end{aligned}
$$

where the $\rho_{i}$ are defined in (9.5). Observe that $q_{-}^{(f t)}(\omega)$ depends on $E^{t}$ only through the quantities $E_{+}^{t}\left(-i \alpha_{i}\right)$ so that it is a state variable, as noted in Sec. 10 and as must be the case by Corollary 9.2. Also, from (11.7)

$$
\begin{aligned}
E_{f}^{t}(\omega) & =i \sum_{l=1}^{n} \frac{B_{l}^{(f t)}}{\omega+i \rho_{l}}, \\
B_{l}^{(f t)} & =\sum_{i=1}^{n} R_{i}^{f} K_{i l}^{f} E_{+}^{t}\left(-i \alpha_{i}\right), \\
K_{i l}^{f} & =\frac{\prod_{\substack{j=1 \\
j \neq i}}^{n}\left(\rho_{l}-\alpha_{j}\right)}{\prod_{\substack{j=1 \\
j \neq l}}^{n}\left(\rho_{l}-\rho_{j}\right)} .
\end{aligned}
$$


Recalling (11.8) we see that

$$
\begin{aligned}
& E_{f-}^{t}(\omega)=-\frac{B_{1-}^{(f t)}}{i \omega^{+}}+i \sum_{\rho_{l}>0} \frac{B_{l}^{(f t)}}{\omega+i \rho_{l}}, \\
& E_{f+}^{t}(\omega)=-\frac{B_{1+}^{(f t)}}{i \omega^{-}}+i \sum_{\rho_{l}<0} \frac{B_{l}^{(f t)}}{\omega+i \rho_{l}},
\end{aligned}
$$

where $B_{1 \pm}^{(f t)}$ corresponds to $\rho_{1}=\gamma_{1}=0$. In the time domain, we have

$$
\begin{aligned}
& E_{f o-}^{t}(s)=B_{1-}^{(f t)}+\sum_{\rho_{l}>0} B_{l}^{(f t)} e^{\rho_{l} s}, \quad s<0, \\
& E_{f o+}^{t}(s)=-B_{1+}^{(f t)}-\sum_{\rho_{l}<0} B_{l}^{(f t)} e^{\rho_{l} s}, \quad s>0 .
\end{aligned}
$$

We know from (11.17) that $E_{f o+}^{t}\left(0^{+}\right) \neq E_{f o-}^{t}\left(0^{-}\right)$. Equations (11.16) and (11.17) are identities obeyed by the coefficients $B_{1 \pm}^{(f t)}$ and $B_{l}^{(f t)}$. In order to determine $B_{1+}^{(f t)}$ and $B_{1-}^{(f t)}$ separately, one must impose $(11.18)$ in intermediate cases.

The quantity $K_{f}(t)$, given by $(9.8)$, has the form

$$
K_{f}(t)=-h_{\infty} \sum_{i=1}^{n} \frac{R_{i}^{f}}{\alpha_{i}} e_{i}(t),
$$

where $e_{i}(t)$ is defined by (10.1).

Using (12.1), we find that the free energy $\psi_{f}(t)$, given by (11.6), has the form [4]

$$
\begin{aligned}
\psi_{f}(t) & =S(t)+H_{\infty} \sum_{i, j=1}^{n} \frac{R_{i}^{f} R_{j}^{f}}{\alpha_{i}+\alpha_{j}} E_{+}^{t}\left(-i \alpha_{i}\right) E_{+}^{t}\left(-i \alpha_{j}\right) \\
& =S(t)+\frac{1}{2} \int_{0}^{\infty} d s_{1} \int_{0}^{\infty} d s_{2} E^{t}\left(s_{1}\right) F_{f}\left(s_{1}, s_{2}\right) E^{t}\left(s_{2}\right), \\
F_{f}\left(s_{1}, s_{2}\right) & =2 H_{\infty} \sum_{i, j=1}^{n} \frac{R_{i}^{f} R_{j}^{f}}{\alpha_{i}+\alpha_{j}} e^{-\alpha_{i} s_{1}-\alpha_{j} s_{2}} .
\end{aligned}
$$

The first relation can be recast in the form (10.2) with

$$
C_{i j}^{f}=2 H_{\infty} \frac{R_{i}^{f} R_{j}^{f}}{\left(\alpha_{i}+\alpha_{j}\right) \alpha_{i} \alpha_{j}} .
$$

This follows by the argument used in [4] with $R_{i}^{f}$ replacing $R_{i}$ since the quantities $R_{i}^{f}$ obey the required identities proved for $R_{i}$ in that reference. These identities are necessary to prove (10.3). The matrix defined by (12.3) is not manifestly positive definite. However, comparing with (11.6), we see that it must have this property.

The fact that $\psi_{f}$ is a state variable, deduced earlier by a general argument, is confirmed explicitly by the fact that it can be cast in the form (10.2).

A particular ranking among the free energies will now be established. We start from the minimum energy $\psi_{m}(t)$ which corresponds to $\varepsilon_{i}=1, i=1,2, \ldots, n$. Interchanging a 
zero, $\gamma_{l}, l \neq 1$ transforms the quantities $R_{i}=R_{i}^{(0)}$ as follows:

$$
R_{i}^{(0)} \rightarrow R_{i}^{(1)}=-R_{i}^{(0)}\left\{\frac{\gamma_{l}+\alpha_{i}}{\gamma_{l}-\alpha_{i}}\right\},
$$

where the new factorization is labelled "1". Comparing the matrices $\mathbf{C}^{(1)}$ and $\mathbf{C}^{(0)}$ as defined by (12.3), we find that

$$
\begin{aligned}
{\left[\mathbf{C}^{(1)}-\mathbf{C}^{(0)}\right]_{i j} } & =\mathbf{C}_{i j}^{(0)}\left\{\left[\frac{\gamma_{l}+\alpha_{i}}{\gamma_{l}-\alpha_{i}}\right]\left[\frac{\gamma_{l}+\alpha_{j}}{\gamma_{l}-\alpha_{j}}\right]-1\right\} \\
& =2 \gamma_{l} \mathbf{C}_{i j}^{(0)} \frac{\left(\alpha_{i}+\alpha_{j}\right)}{\left(\gamma_{l}-\alpha_{i}\right)\left(\gamma_{l}-\alpha_{j}\right)} \\
& =2 \frac{\gamma_{l} R_{i}^{(0)} R_{j}^{(0)}}{\left(\gamma_{l}-\alpha_{i}\right)\left(\gamma_{l}-\alpha_{j}\right)}
\end{aligned}
$$

which, leaving aside the positive quantitity $2 \gamma_{l}$, is the outer product of a vector with itself and if inserted into a quadratic form, produces a positive, semi-definite result. Thus

$$
\psi^{(1)}(t) \geq \psi^{(0)}(t)
$$

If another zero is interchanged, one can show by the same argument that $\psi^{(2)}(t) \geq \psi^{(1)}(t)$ and so on. There are $(n-1)$ ! pathways starting with $\psi_{m}(t)$ and ending with $\psi^{(M)}(t)$ with all zeros interchanged, where, on each step, a zero untouched in previous steps, is interchanged. Along a particular pathway, we have

$$
\psi^{(M)}(t)=\psi^{(n)}(t) \geq \psi^{(n-1)}(t) \geq \cdots \geq \psi^{(1)}(t) \geq \psi^{(0)}(t)=\psi_{m}(t) .
$$

The superscripts $n, n-1$, etc. will have quite different meanings on different pathways. Free energies that lie on different pathways may well be rankable by virtue of more subtle inequalities.

The minimum and maximum free energies in each sequence (12.4) are unique however. These, and quantities associated with them are obtained from the formulae in this section by putting $\rho_{i}=\gamma_{i}, i=2,3, \ldots, n$ for the minimum and $\rho_{i}=-\gamma_{i}, i=2,3, \ldots, n$ for the maximum free energies.

Thus, $\psi_{m}, \psi^{(M)}$ are respectively the minimum and maximum free energies corresponding to the element $\sigma_{R} \in \Sigma_{R}$ defined by $\left(E(t), E^{t}\right)$.

The dissipation function corresponding to $\psi_{f}(t)$ is given by

$$
D_{f}(t)=K_{f}^{2}(t)
$$

where $K_{f}(t)$ is defined by (12.2). These quantities cannot be ranked in the manner of (12.4).

The $N$ free energies defined by different factorizations are all in the set $\mathcal{S}_{\sigma}$ defined in Sec. 10. They are in fact on the boundary of this convex set, in a sense that will now be discussed.

From Sec. 10 we see that the boundary of the convex set $\mathcal{S}_{\sigma}$ will be indicated by the breakdown in the positive definiteness of either $\mathbf{C}$ or $\Gamma$ or both. From (12.2) and (12.5), it is clear that

$$
\Gamma_{i j}^{(f)}=2 H_{\infty} \frac{R_{i}^{f}}{\alpha_{i}} \frac{R_{j}^{f}}{\alpha_{j}}
$$


which is a positive semi-definite matrix. Thus we are on the boundary of $\mathcal{S}_{\sigma}$ in the sense that the positive definiteness of $\Gamma^{f}$ is breaking down to positive semi-definiteness. This process is best pictured by considering the diagonalized form of $\Gamma^{f}$. In general, all the eigenvalues will be positive, corresponding to positive definiteness. When one or more eigenvalues become zero, we have positive semi-definiteness. When any cigenvalue becomes zero, we are on the boundary of $\mathcal{S}_{\sigma}$, because if it becomes negative, however slightly, the matrix is of indefinite sign.

Now, relation (12.6) corresponds to all eigenvalues being zero but one. The vector $\mathbf{R}^{(f)}$ with components $\frac{R_{i}^{f}}{\alpha_{i}}$ is clearly an eigenvector of $\Gamma^{f}$ with eigenvalue

$$
\lambda^{f}=2 H_{\infty} \sum_{i=1}^{n}\left[\frac{R_{i}^{f}}{\alpha_{i}}\right]^{2} .
$$

Acting on the $(n-1)$-dimensional subspace orthogonal to $\mathbf{R}^{(f)}$, it gives zero.

The convexity of $\mathcal{S}_{\sigma}$ means that we can form a family of free energies given by

$$
\psi(t)=\sum_{f} a_{f}^{2} \psi_{f}(t), \quad \sum_{f} a_{f}^{2}=1,
$$

where the sum is in general over all $2^{n-1}$ factorizations and each $a_{f}$ can take all real values. Clearly $\psi_{m}(t) \leq \psi(t) \leq \psi^{(M)}(t)$.

For $n=1$, the set $\mathcal{S}_{\sigma}$ reduces to a singleton-as demonstrated for a very general exponential form in [9]. For $n=2$, the range of allowable $\mathbf{C}$ and $\Gamma$ matrices can be determined by elementary considerations. This was done by Breuer and Onat [21]. Their results agree with the general formulae developed here. Note that, in this case, an explicit formula can be given for the quantity $\gamma_{2}$.

\section{REFERENCES}

[1] W. Noll, A new mathematical theory of simple materials, Arch. Rational Mech. Anal. 48, 1-50 (1972)

[2] B.D. Coleman and D.R. Owen, A mathematical foundation for thermodynamics, Arch. Rational Mech. Anal. 54, 1-104 (1974)

[3] M. Fabrizio, C. Giorgi, and A. Morro, Free energies and dissipation properties for systems with memory, Arch. Rational Mech. Anal. 125, 341-373 (1994)

[4] J. M. Golden, Free energies in the frequency domain: The scalar case, Quart. Appl. Math. 58, $127-150$ (2000)

[5] L. Deseri, G. Gentili, and J.M. Golden, An explicit formula for the minimum free energy in linear viscoelasticity, J. Elasticity 54, 141-185 (1999)

[6] W. A. Day, The thermodynamics of materials with memory, Materials with Memory, D. Graffi ed., Liguori, Napoli, 1979

[7] M. Fabrizio and A. Morro, Mathematical Problems in Linear Viscoelasticity, SIAM, Philadelphia, 1992

[8] G. Del Piero and L. Deseri, On the concepts of state and free energy in linear viscoelasticity, Arch. Rational Mech. Anal. 138, 1-35 (1997)

[9] G. Del Piero and L. Deseri, On the analytic expression of the free energy in linear viscoelasticity, J. Elasticity 43, 247-278 (1996)

[10] S. Breuer and E.T. Onat, On recoverable work in linear viscoelasticity, Z. Angew. Math. Phys. 15, 13-21 (1964)

[11] V. Volterra, Theory of functional and of integral and integro-differential equations, Blackie and Son Limited, London (1930) 
[12] W. A. Day, Reversibility, recoverable work and free energy in linear viscoelasticity, Quart. J. Mech. Appl. Math. 23, 1-15 (1970)

[13] M. Fabrizio and A. Morro, Viscoelastic relaxation functions compatible with thermodynamics, J. Elasticity 19, 63-75 (1988)

[14] N. I. Muskhelishvili, Singular Integral Equations. Boundary Problems of Function Theory and their Application to Mathematical Physics, Noordhoff, Groningen, 1953

[15] J.M. Golden and G.A.C. Graham, Boundary Value Problems in Linear Viscoelasticity, SpringerVerlag, Berlin, 1988

[16] B.D. Coleman, Thermodynamics of materials with memory, Arch. Rational Mech. Anal. 17, 1-45 (1964)

[17] B.D. Coleman and V.J. Mizel, A general theory of dissipation in materials with memory, Arch. Rational Mech. Anal. 27, 255-274 (1967)

[18] D. Graffi and M. Fabrizio, Sulla nozione di stato per materiali viscoelastici di tipo "rate", Atti Acc. Naz. Lincei 83, 201-208 (1990)

[19] D. Graffi and M. Fabrizio, Non unicità dell'energia libera per i materiali viscoelastici, Atti Acc. Naz. Lincei 83, 209-214 (1990)

[20] M. Fabrizio, C. Giorgi, and A. Morro, Internal dissipation, relaxation property and free energy in materials with fading memory, J. Elasticity 40, 107-122 (1995)

[21] S. Breuer and E.T. Onat, On the determination of free energy in viscoelastic solids, Z. Angew. Math. Phys. 15, 185-191 (1964) 